\title{
22. PALEOMAGNETISM OF SPECIMENS FROM LEG 3 OF THE DEEP SEA DRILLING PROJECT ${ }^{1}$
}

\author{
K. W. Henry and N. D. Opdyke \\ Lamont Doherty Geological Observatory of Columbia University, Palisades, New York
}

Leg 3 of the Deep Sea Drilling Project provided the first opportunity to look at actual sections of paleomag. netic stratigraphy for parts of the Lower Miocene, Upper Oligocene and the Cretaceous-Tertiary boundary. This was because of the much improved core recovery which included several long sections of continuous core, and to a greater density of samples than was recovered from either Leg 1 or 2.

Samples were treated in the same manner as those for the two previous legs. All samples were measured at the natural remanent magnetization (NRM), and were cleaned in a field of 50 oersteds. The summary data is given in Table 1 .

An A-F demagnetization curve for fields up to 200 oersteds was plotted on a pilot sample from each hole. These curves can be seen in Figures 1 through 13. Most samples that are of easily measureable intensity are seen to be partially stable. Sample 3-13A-5-1, however, is extremely weak $\left(10^{-8} \mathrm{emu} / \mathrm{gm}\right)$, which probably is the reason for the strange behavior of this sample.

Figures 14 through 20 illustrate attempts to form a magnetic stratigraphy for the more densely sampled sections. Normal sections (which have a negative inclination for the southern latitude Sites 14 through 21) are indicated in black and reversed sections are shown in diagonal strips. The sections that were not cored are white. Actual sample points are indicated by arrows to the left of each diagram.

Problems in correlation occur both within individual sites and between different sites. In two sites (Sites 15 and 19) paleontologists recognized the problem of repeated sections found in sequential cores. Thus the paleomagnetic data from Hole 15, Core 9, Hole 19, Core 8 and Hole 19, Core 9 should be disregarded.

Another problem occurs in correlations between adjacent holes at the same site. In Site 20, for instance, the Upper Oligocene sections of Hole 20B and Hole 20C

\footnotetext{
${ }^{1}$ Lamont-Doherty Geological Observatory of Columbia University Contribution No. 1346.
}

seem to be out of phase. In Site 17, although the polarity overlap of Holes 17A and 17B in the Upper Oligocene seems pretty good, the inclination values are quite different. In Hole 17A, Core 4 (from 96 to 103 meters) the inclinations are extremely high-from 65 to 75 degrees-while in Hole 17B, Core 1 and 2, the inclination values are low.

Correlation between sites is extremely difficult due to the widely differing sedimentation rates and imprecise dating. An attempt can be made to correlate only when a distinct paleontological boundary or a distinct, synchronous lithologic unit was cored more than once. There are three opportunities present: the Upper Oligocene-Lower Oligocene boundary in Sites 19 and 20, the Eocene-Lower Oligocene boundary in Sites 14 and 19; and, several corings of the Maxwell Chalk Layer.

The Lower Oligocene-Upper Eocene boundary occurs at Site 19 at 79 meters and at Site 14 at the very bottom of the hole. In both cases the boundary occurs in a reversely magnetized section of core with a normally magnetized section above. The Upper Oligocene/Lower Oligocene boundary in Hole 14 at 87 meters and Hole $20 \mathrm{~A}$ at 18 meters also occurs in reversely magnetized sediments. Thus in both of these cases the paleontology and paleomagnetics are mutually supporting.

The Maxwell Chalk occurs in six separate holes. In Holes 17B, 20B and 22 it occurs in a normally magnetized section of core; while at Holes 14,17A and 19, it occurs in reversely magnetized sections of core. The reversed section at Hole 14 rests on only one measurement, and in Hole 17A on two measurements. The sampled sections in both of these holes are short. In only Hole 19 is the reversely magnetized section of core associated with the Maxwell Chalk well established, and in this hole the chalk is .5 centimeter thick so that miscorrelation is possible. The most reliable measurements are probably from Site 22 where a thick section of chalk was cored. Here a normal direction is observed. Therefore, the most reasonable interpretation is that the magnetic field of the earth was normal at the time of deposition of the Maxwell Chalk. This interpretation, however, is by no means unambiguous.

The other significant paleontological boundary that was cored during Leg 3 was the Cretaceous-Tertiary 
boundary seen in Hole 20C. The extremely close sampling interval here allows for a well documented view across the boundary which seems to have occurred at a time when the magnetic field of the earth was reversed. There are two anomalous, normally magnetized samples that occur in the section. The first-20C-6-4, 112 centimeters-is a sample of a reworked Cretaceous clump which is found in Paleocene sediments just above the boundary; and, the other-20C-6-5, $0-2$ centimeters -is taken from the very top of a section and thus is very possibly disturbed. Although based on only one section of core, it seems most likely that the
Cretaceous-Tertiary boundary occurred while the field of the earth remained reversed.

The outstanding results of the Leg 3 paleomagnetic studies can be summarized as follows:

1. Sediments of both polarities occur in all the geological epochs of the Cenozoic and into the Upper Cretaceous.

2. The Upper Oligocene/Lower Oligocene boundary, the Oligocene/Eocene boundary and the Tertiary/Cretaceous boundary occur at times in which the magnetic field of the earth was reversed. 
TABLE 1

Summary of Magnetic Data

The data are presented in order by site. Sample numbers are given as hole number, core, section, and then the starting depth of the sample in centimeters.

Magnetic directions and intensities in emu/gm are given for each sample at NRM, and followed by the A-F demagnetization in a field of 50 oersteds. Note that a negative (positive) inclination indicates a normal (reversed) field direction for sites in the southern hemisphere (Sites 14 through 22); the opposite is true for Site 13 in the northern hemisphere.

An asterisk beside a sample number indicates that it is used as a pilot sample for the site and an A-F demagnetization curve has been completed. These are presented in Figures 1 through 13.

\begin{tabular}{|c|c|c|c|c|c|c|c|c|c|}
\hline \multirow[b]{2}{*}{ Hole } & \multirow[b]{2}{*}{ Core } & \multirow[b]{2}{*}{ Section } & \multirow[b]{2}{*}{$\begin{array}{l}\text { Sampled at } \\
\text { (cm) }\end{array}$} & \multicolumn{3}{|c|}{ NRM } & \multicolumn{3}{|c|}{50 oersted } \\
\hline & & & & Decl. & Incl. & $\begin{array}{l}\text { Intensity } \\
\text { emu/gm }\end{array}$ & Decl. & Incl. & $\begin{array}{l}\text { Intensity } \\
\text { emu/gm }\end{array}$ \\
\hline $13 \mathrm{~A}$ & 2 & 1 & 114 & 202.7 & -68.8 & $0.1136 \times 10^{-6}$ & 56.3 & -78.6 & $0.6949 \times 10^{-7}$ \\
\hline $13 \mathrm{~A}$ & 3 & 1 & 113 & 132.1 & -1.3 & $0.3500 \times 10^{-6}$ & 123.5 & 67.1 & $0.9303 \times 10^{-6}$ \\
\hline $13 \mathrm{~A}$ & 3 & 2 & 11 & 225.7 & 4.4 & $0.3656 \times 10^{-6}$ & 208.1 & 25.2 & $0.2566 \times 10^{-6}$ \\
\hline $13 \mathrm{~A}$ & 3 & 2 & 50 & 241.1 & -23.8 & $0.2675 \times 10^{-6}$ & 223.6 & -21.5 & $0.3069 \times 10^{-6}$ \\
\hline $13 \mathrm{~A}$ & 3 & 4 & 50 & 215.7 & -5.9 & $0.2919 \times 10^{-6}$ & 63.9 & 29.1 & $0.3174 \times 10^{-6}$ \\
\hline $13 \mathrm{~A}$ & 5 & 1 & $49-51$ & 222.7 & -36.3 & $0.8200 \times 10^{-7}$ & 240.6 & -37.3 & $0.8616 \times 10^{-7}$ \\
\hline $14 \mathrm{~A}$ & 1 & 1 & 99 & 192.3 & -11.3 & $0.5723 \times 10^{-5}$ & 218.4 & -40.3 & $0.1726 \times 10^{-5}$ \\
\hline $14 \mathrm{~A}$ & 1 & 3 & $50-2$ & 192.1 & 26.4 & $0.6244 \times 10^{-5}$ & 188.9 & 30.4 & $0.2046 \times 10^{-5}$ \\
\hline $14 \mathrm{~A}$ & 1 & 5 & 39 & 171.5 & -9.6 & $0.8470 \times 10^{-5}$ & 161.8 & -21.1 & $0.2174 \times 10^{-5}$ \\
\hline $14 \mathrm{~A}$ & 1 & 6 & 49 & 175.2 & 13.6 & $0.6781 \times 10^{-5}$ & 207.0 & 54.6 & $0.2915 \times 10^{-5}$ \\
\hline 14 & 2 & 1 & 120 & 170.3 & 10.1 & $0.4595 \times 10^{-5}$ & 171.4 & -12.0 & $0.1486 \times 10^{-5}$ \\
\hline 14 & 2 & 2 & 48 & 169.0 & 1.3 & $0.2717 \times 10^{-5}$ & 162.9 & -11.8 & $0.8346 \times 10^{-6}$ \\
\hline 14 & 2 & 3 & 65 & 44.3 & -25.9 & $0.1829 \times 10^{-4}$ & 31.3 & -19.0 & $0.1841 \times 10^{-4}$ \\
\hline 14 & 2 & 4 & 50 & 203.6 & -14.4 & $0.4833 \times 10^{-5}$ & 235.5 & -28.5 & $0.2000 \times 10^{-5}$ \\
\hline 14 & 2 & 5 & 52 & 216.2 & -7.0 & $0.3208 \times 10^{-5}$ & 246.6 & -15.1 & $0.1809 \times 10^{-5}$ \\
\hline 14 & 3 & 2 & 52 & 188.1 & -34.5 & $0.3942 \times 10^{-5}$ & 207.4 & -54.7 & $0.2064 \times 10^{-5}$ \\
\hline
\end{tabular}


TABLE 1-Continued

\begin{tabular}{|c|c|c|c|c|c|c|c|c|c|}
\hline \multirow[b]{2}{*}{ Hole } & \multirow[b]{2}{*}{ Core } & \multirow[b]{2}{*}{ Section } & \multirow[b]{2}{*}{$\begin{array}{l}\text { Sampled at } \\
(\mathrm{cm})\end{array}$} & \multicolumn{3}{|c|}{ NRM } & \multicolumn{3}{|c|}{50 oersted } \\
\hline & & & & Decl. & Incl. & $\begin{array}{l}\text { Intensity } \\
\text { emu/gm }\end{array}$ & Decl. & Incl. & $\begin{array}{l}\text { Intensity } \\
\text { emu/gm }\end{array}$ \\
\hline 14 & 3 & 3 & 50 & 196.1 & 7.5 & $0.3763 \times 10^{-5}$ & 148.0 & 6.3 & $0.1195 \times 10^{-5}$ \\
\hline 14 & 3 & 4 & 50 & 166.6 & -10.0 & $0.3661 \times 10^{-5}$ & 181.1 & -14.6 & $0.1023 \times 10^{-5}$ \\
\hline 14 & 4 & 1 & 36 & 105.5 & 6.5 & $0.6383 \times 10^{-5}$ & 106.4 & 5.4 & $0.3801 \times 10^{-5}$ \\
\hline 14 & 5 & 2 & 100 & 170.7 & -22.2 & $0.2399 \times 10^{-5}$ & 137.0 & -30.4 & $0.1106 \times 10^{-5}$ \\
\hline 14 & 5 & 3 & 56 & 201.7 & 25.9 & $0.1254 \times 10^{-5}$ & 286.1 & -20.8 & $0.4556 \times 10^{-6}$ \\
\hline 14 & 5 & 4 & 50 & 222.9 & 31.1 & $0.1960 \times 10^{-5}$ & 259.3 & 54.0 & $0.1136 \times 10^{-5}$ \\
\hline 14 & 6 & 3 & 50 & 165.0 & 15.8 & $0.1833 \times 10^{-5}$ & 148.1 & 47.7 & $0.8679 \times 10^{-6}$ \\
\hline 14 & 6 & 4 & 50 & 195.3 & 15.0 & $0.1824 \times 10^{-5}$ & 210.8 & 75.7 & $0.1535 \times 10^{-5}$ \\
\hline 14 & 6 & 5 & 50 & 177.0 & 53.9 & $0.3287 \times 10^{-5}$ & 213.0 & 67.5 & $0.2231 \times 10^{-5}$ \\
\hline 14 & 6 & 6 & 50 & 171.6 & 12.6 & $0.2329 \times 10^{-5}$ & 143.7 & 30.0 & $0.8088 \times 10^{-6}$ \\
\hline 14 & 7 & 1 & 50 & 145.7 & 42.4 & $0.3654 \times 10^{-5}$ & 122.4 & 47.7 & $0.2965 \times 10^{-5}$ \\
\hline 14 & 7 & 2 & 50 & 297.2 & 45.4 & $0.4576 \times 10^{-5}$ & 321.7 & 37.8 & $0.3790 \times 10^{-5}$ \\
\hline 14 & 7 & 3 & 50 & 189.2 & 19.7 & $0.2781 \times 10^{-5}$ & 193.8 & 52.0 & $0.8881 \times 10^{-6}$ \\
\hline 14 & 7 & 4 & 50 & 154.2 & -20.8 & $0.2041 \times 10^{-5}$ & 64.5 & 58.1 & $0.1508 \times 10^{-5}$ \\
\hline 14 & 7 & 5 & 100 & 156.0 & 26.3 & $0.2820 \times 10^{-5}$ & 117.6 & 16.8 & $0.1561 \times 10^{-5}$ \\
\hline 14 & 7 & 6 & 9 & 145.5 & 32.2 & $0.6908 \times 10^{-5}$ & 142.1 & 42.3 & $0.5191 \times 10^{-5}$ \\
\hline 14 & 8 & 1 & 45 & 340.8 & 46.5 & $0.1979 \times 10^{-3}$ & 344.3 & 46.3 & $0.1674 \times 10^{-3}$ \\
\hline 14 & 8 & 5 & 50 & 198.6 & 52.3 & - & 182.2 & 53.7 & - \\
\hline 14 & 9 & 2 & 50 & 301.1 & -10.0 & $0.3738 \times 10^{-3}$ & 303.3 & -0.0 & $0.3353 \times 10^{-3}$ \\
\hline 14 & 9 & 4 & 100 & 172.5 & -10.1 & $0.2732 \times 10^{-3}$ & 210.7 & -48.3 & $0.1513 \times 10^{-5}$ \\
\hline 14 & 9 & 5 & 50 & 253.8 & -32.8 & $0.2017 \times 10^{-5}$ & 252.4 & -52.5 & $0.8186 \times 10^{-6}$ \\
\hline 14 & 9 & 5 & 123 & 172.3 & 36.1 & $0.2369 \times 10^{-5}$ & 119.7 & 10.7 & $0.6354 \times 10^{-6}$ \\
\hline 14 & 9 & 6 & 15 & 141.6 & 32.7 & $0.5742 \times 10^{-5}$ & 133.3 & 40.2 & $0.3818 \times 10^{-5}$ \\
\hline 14 & 9 & 6 & 50 & 116.0 & 35.7 & $0.7914 \times 10^{-5}$ & 109.4 & 44.2 & $0.4906 \times 10^{-5}$ \\
\hline 14 & 9 & 6 & 100 & 163.5 & 26.3 & $0.2195 \times 10^{-5}$ & 103.4 & 66.3 & $0.4901 \times 10^{-6}$ \\
\hline 15 & 1 & 1 & 50 & 139.0 & -68.1 & $0.5962 \times 10^{-5}$ & 142.5 & -73.1 & $0.3079 \times 10^{-5}$ \\
\hline 15 & 1 & 1 & 103 & 170.7 & -28.3 & - & 138.3 & -30.5 & $\longrightarrow$ \\
\hline 15 & 1 & 2 & 50 & 147.2 & -73.2 & $0.2303 \times 10^{-5}$ & 129.5 & -65.9 & $0.1129 \times 10^{-5}$ \\
\hline 15 & 1 & 3 & 54 & 129.3 & -15.7 & $0.3462 \times 10^{-5}$ & 150.0 & -35.1 & $0.2071 \times 10^{-5}$ \\
\hline
\end{tabular}


TABLE 1-Continued

\begin{tabular}{|c|c|c|c|c|c|c|c|c|c|}
\hline \multirow[b]{2}{*}{ Hole } & \multirow[b]{2}{*}{ Core } & \multirow[b]{2}{*}{ Section } & \multirow[b]{2}{*}{$\begin{array}{l}\text { Sampled at } \\
\text { (cm) }\end{array}$} & \multicolumn{3}{|c|}{ NRM } & \multicolumn{3}{|c|}{50 oersted } \\
\hline & & & & Decl. & Incl. & $\begin{array}{l}\text { Intensity } \\
\text { emu/gm }\end{array}$ & Decl. & Incl. & $\begin{array}{l}\text { Intensity } \\
\text { emu/gm }\end{array}$ \\
\hline 15 & 1 & 5 & 33 & 213.5 & 22.9 & $0.5437 \times 10^{-5}$ & 221.7 & 31.3 & $0.3407 \times 10^{-5}$ \\
\hline 15 & 1 & 5 & 54 & 235.9 & 34.0 & $0.1290 \times 10^{-6}$ & 223.2 & 43.1 & $0.8957 \times 10^{-5}$ \\
\hline 15 & 1 & 6 & 18 & 168.6 & -29.5 & $0.2900 \times 10^{-5}$ & 171.8 & -29.8 & $0.1300 \times 10^{-5}$ \\
\hline 15 & 1 & 6 & 50 & 136.5 & 12.7 & $0.1345 \times 10^{-5}$ & 251.1 & -24.2 & $0.7126 \times 10^{-6}$ \\
\hline 15 & 1 & 6 & 71 & 223.7 & -4.1 & & 231.9 & -2.6 & \\
\hline 15 & 2 & 1 & 48 & 271.8 & -9.9 & $0.6661 \times 10^{-5}$ & 273.3 & 54.9 & $0.2453 \times 10^{-5}$ \\
\hline 15 & 2 & 4 & 107 & 208.4 & 17.5 & $0.1980 \times 10^{-5}$ & 213.6 & 60.7 & $0.7028 \times 10^{-6}$ \\
\hline 15 & 2 & 5 & 48 & 78.0 & -47.6 & $0.6320 \times 10^{-5}$ & 60.2 & -50.9 & $0.1492 \times 10^{-5}$ \\
\hline 15 & 2 & 5 & 100 & 142.9 & 25.0 & $\longrightarrow$ & 53.8 & 32.9 & $\longrightarrow$ \\
\hline 15 & 2 & 6 & 48 & 77.1 & -84.6 & $\longrightarrow$ & 66.8 & -48.0 & $\longrightarrow$ \\
\hline 15 & 2 & 6 & 105 & 164.2 & 33.2 & - & 148.1 & 43.3 & $\longrightarrow$ \\
\hline 15 & 3 & 1 & 52 & 269.3 & -51.6 & $0.6200 \times 10^{-5}$ & 266.7 & -55.5 & $0.2573 \times 10^{-5}$ \\
\hline 15 & 3 & 1 & 100 & 173.3 & -56.1 & $0.5342 \times 10^{-5}$ & 168.3 & -13.7 & $0.8882 \times 10^{-6}$ \\
\hline 15 & 3 & 2 & 50 & 263.9 & 46.4 & $0.1257 \times 10^{-4}$ & 275.6 & -47.0 & $0.4048 \times 10^{-5}$ \\
\hline 15 & 3 & 2 & 100 & 173.4 & -31.6 & $0.3896 \times 10^{-5}$ & 199.5 & 3.4 & $0.1382 \times 10^{-5}$ \\
\hline 15 & 3 & 4 & 43 & 93.9 & 26.5 & $\longrightarrow$ & 86.2 & 32.4 & $\longrightarrow$ \\
\hline 15 & 3 & 4 & 100 & 160.8 & 7.1 & $0.1759 \times 10^{-5}$ & 157.9 & 4.6 & $0.3442 \times 10^{-6}$ \\
\hline 15 & 3 & 5 & 100 & 185.0 & 13.7 & $0.1921 \times 10^{-5}$ & 178.5 & 8.4 & $0.8279 \times 10^{-6}$ \\
\hline 15 & 3 & 6 & 9 & 172.7 & -38.5 & - & 162.5 & -45.2 & - \\
\hline 15 & 4 & 1 & 23 & 240.5 & 50.0 & $\longrightarrow$ & 282.9 & 53.7 & $\longrightarrow$ \\
\hline 15 & 4 & 1 & 55 & 65.8 & -29.7 & - & 63.2 & -22.2 & $\longrightarrow$ \\
\hline 15 & 4 & 1 & 98 & 202.4 & 37.4 & - & 119.4 & 78.0 & $\longrightarrow$ \\
\hline 15 & 4 & 2 & 50 & 170.6 & -20.1 & $0.3198 \times 10^{-5}$ & 162.9 & 18.4 & $0.9421 \times 10^{-6}$ \\
\hline 15 & 4 & 2 & 102 & 148.3 & 40.6 & $0.7453 \times 10^{-6}$ & 72.4 & 31.7 & $0.5487 \times 10^{-6}$ \\
\hline 15 & 4 & 3 & 12 & 164.0 & 16.9 & $0.9072 \times 10^{-6}$ & 22.1 & 43.4 & $0.5503 \times 10^{-6}$ \\
\hline$* 15$ & 4 & 3 & $41-3$ & 143.9 & 10.4 & $0,7885 \times 10^{-6}$ & 70.2 & 36.9 & $0.4740 \times 10^{-6}$ \\
\hline 15 & 4 & 4 & 54 & 173.9 & 0.5 & $0.3061 \times 10^{-5}$ & 174.7 & 9.7 & $0.1264 \times 10^{-5}$ \\
\hline 15 & 4 & 4 & 100 & 211.6 & -17.8 & $0.1092 \times 10^{-5}$ & 66.8 & -65.4 & $0.4916 \times 10^{-6}$ \\
\hline 15 & 4 & 5 & 12 & 130.7 & 13.1 & $0.1017 \times 10^{-5}$ & 185.1 & -8.4 & $0.4905 \times 10^{-6}$ \\
\hline
\end{tabular}


TABLE 1-Continued

\begin{tabular}{|c|c|c|c|c|c|c|c|c|c|}
\hline \multirow[b]{2}{*}{ Hole } & \multirow[b]{2}{*}{ Core } & \multirow[b]{2}{*}{ Section } & \multirow[b]{2}{*}{$\begin{array}{l}\text { Sampled at } \\
(\mathrm{cm})\end{array}$} & \multicolumn{3}{|c|}{ NRM } & \multicolumn{3}{|c|}{50 oersted } \\
\hline & & & & Decl. & Incl. & $\begin{array}{l}\text { Intensity } \\
\text { emu/gm }\end{array}$ & Decl. & Incl. & $\begin{array}{l}\text { Intensity } \\
\text { emu/gm }\end{array}$ \\
\hline 15 & 4 & 5 & 100 & 236.3 & 38.4 & $0.5856 \times 10^{-6}$ & 76.1 & -1.0 & $0.4802 \times 10^{-6}$ \\
\hline 15 & 4 & 6 & 12 & 241.1 & -13.2 & $0.2963 \times 10^{-5}$ & 281.8 & -16.3 & $0.6265 \times 10^{-6}$ \\
\hline 15 & 4 & 6 & 53 & 203.5 & 2.2 & $0.5402 \times 10^{-5}$ & 211.7 & -6.6 & $0.1770 \times 10^{-5}$ \\
\hline 15 & 4 & 6 & 100 & 230.9 & -8.0 & $\longrightarrow$ & 204.3 & -18.5 & - \\
\hline 15 & 5 & 1 & 11 & 193.0 & -67.9 & $0.1452 \times 10^{-4}$ & 236.7 & -10.9 & $0.6899 \times 10^{-6}$ \\
\hline 15 & 5 & 1 & 60 & 196.6 & 2.3 & $0.1349 \times 10^{-4}$ & 176.5 & 44.5 & $0.3330 \times 10^{-5}$ \\
\hline 15 & 5 & 1 & 100 & 228.7 & -6.2 & $0.6351 \times 10^{-5}$ & 305.2 & 18.0 & $0.1089 \times 10^{-5}$ \\
\hline 15 & 5 & 2 & 45 & 169.4 & -11.0 & $0.3486 \times 10^{-5}$ & 206.4 & -0.8 & $0.2172 \times 10^{-5}$ \\
\hline 15 & 5 & 2 & 100 & 197.4 & -14.1 & $0.3478 \times 10^{-5}$ & 120.7 & 21.8 & $0.1192 \times 10^{-5}$ \\
\hline 15 & 5 & 3 & 12 & 194.2 & -14.0 & - & 85.3 & 12.6 & $\longrightarrow$ \\
\hline 15 & 5 & 3 & 52 & 192.5 & -14.3 & $0.4447 \times 10^{-5}$ & 259.0 & -5.3 & $0.1787 \times 10^{-5}$ \\
\hline 15 & 5 & 3 & 100 & 169.3 & 0.9 & $0.2689 \times 10^{-5}$ & - & $\longrightarrow$ & \\
\hline 15 & 5 & 4 & 9 & 205.4 & -16.9 & $0.3224 \times 10^{-5}$ & 188.0 & -1.9 & $0.1113 \times 10^{-5}$ \\
\hline 15 & 5 & 4 & 48 & 159.8 & 48.9 & $0.5072 \times 10^{-5}$ & 142.9 & 22.7 & $0.1277 \times 10^{-5}$ \\
\hline 15 & 5 & 4 & 100 & 233.1 & 48.8 & $0.2788 \times 10^{-5}$ & 58.2 & 62.6 & $0.1553 \times 10^{-5}$ \\
\hline 15 & 5 & 5 & 11 & 142.9 & -26.0 & - & - & - & $\longrightarrow$ \\
\hline 15 & 5 & 5 & 51 & 249.3 & 32.2 & $0.2035 \times 10^{-4}$ & 257.7 & 53.4 & $0.3924 \times 10^{-5}$ \\
\hline 15 & 5 & 6 & 11 & 194.4 & 10.5 & $0.3200 \times 10^{-5}$ & 121.7 & 89.5 & $0.1193 \times 10^{-3}$ \\
\hline 15 & 5 & 6 & 100 & 162.0 & 57.1 & $\longrightarrow$ & 291.5 & 66.4 & $\longrightarrow$ \\
\hline 15 & 6 & 1 & 11 & 212.8 & 12.8 & $0.1550 \times 10^{-4}$ & 247.3 & 39.9 & $0.5862 \times 10^{-5}$ \\
\hline 15 & 6 & 1 & 67 & 201.5 & -7.4 & $0.1839 \times 10^{-4}$ & 180.3 & 45.4 & $0.5720 \times 10^{-5}$ \\
\hline 15 & 6 & 2 & 62 & 191.1 & 15.7 & $\longrightarrow$ & 163.2 & 15.4 & $\longrightarrow$ \\
\hline 15 & 6 & 3 & 2 & 190.9 & 20.7 & $0.2669 \times 10^{-4}$ & 105.9 & 23.1 & $0.2289 \times 10^{-5}$ \\
\hline 15 & 6 & 3 & 11 & 166.0 & -59.9 & $0.2000 \times 10^{-4}$ & 212.9 & 8.5 & $0.2619 \times 10^{-5}$ \\
\hline 15 & 6 & 3 & 52 & 210.1 & -16.8 & $0.2010 \times 10^{-4}$ & 140.1 & 17.8 & $0.4387 \times 10^{-5}$ \\
\hline 15 & 6 & 3 & 100 & 214.9 & -31.1 & $0.1350 \times 10^{-4}$ & 172.7 & 13.2 & $0.3639 \times 10^{-5}$ \\
\hline 15 & 6 & 4 & 2 & 194.7 & 19.2 & $0.3250 \times 10^{-5}$ & - & - & $\longrightarrow$ \\
\hline 15 & 6 & 4 & 11 & 155.9 & 30.1 & $0.1027 \times 10^{-4}$ & 155.4 & 55.4 & $0.2611 \times 10^{-5}$ \\
\hline 15 & 6 & 4 & 52 & 196.7 & 11.4 & $0.1907 \times 10^{-4}$ & 210.0 & 9.6 & $0.4763 \times 10^{-5}$ \\
\hline
\end{tabular}


TABLE 1-Continued

\begin{tabular}{|c|c|c|c|c|c|c|c|c|c|}
\hline \multirow[b]{2}{*}{ Hole } & \multirow[b]{2}{*}{ Core } & \multirow[b]{2}{*}{ Section } & \multirow[b]{2}{*}{$\begin{array}{l}\text { Sampled at } \\
(\mathrm{cm})\end{array}$} & \multicolumn{3}{|c|}{ NRM } & \multicolumn{3}{|c|}{50 oersted } \\
\hline & & & & Decl. & Incl. & $\begin{array}{l}\text { Intensity } \\
\text { emu/gm }\end{array}$ & Decl. & Incl. & $\begin{array}{l}\text { Intensity } \\
\text { emu/gm }\end{array}$ \\
\hline 15 & 6 & 4 & 101 & 156.2 & -12.3 & $0.9342 \times 10^{-5}$ & 220.1 & -29.1 & $0.2330 \times 10^{-5}$ \\
\hline 15 & 6 & 4 & 108 & 328.3 & -67.7 & $0.2256 \times 10^{-5}$ & 202.3 & -9.2 & $0.3706 \times 10^{-5}$ \\
\hline 15 & 6 & 5 & 11 & 165.1 & 65.2 & $0.4214 \times 10^{-5}$ & 80.7 & 61.4 & $0.2841 \times 10^{-5}$ \\
\hline 15 & 6 & 5 & 51 & 196.6 & 19.2 & $0.5663 \times 10^{-5}$ & 168.2 & 52.8 & $0.2725 \times 10^{-5}$ \\
\hline 15 & 6 & 5 & 100 & 184.7 & -4.9 & $0.4503 \times 10^{-5}$ & 14.7 & -82.1 & $0.1373 \times 10^{-5}$ \\
\hline 15 & 6 & 6 & 2 & 223.0 & -34.3 & $0.7748 \times 10^{-5}$ & 252.1 & -77.1 & $0.6524 \times 10^{-5}$ \\
\hline 15 & 6 & 6 & 10 & 145.9 & 49.2 & $0.6753 \times 10^{-5}$ & 120.0 & 64.8 & $0.3303 \times 10^{-5}$ \\
\hline 15 & 6 & 6 & 52 & 182.6 & -23.2 & $0.6837 \times 10^{-5}$ & 200.2 & -15.9 & $0.2396 \times 10^{-5}$ \\
\hline 15 & 6 & 6 & 100 & 186.8 & 21.5 & $0.7264 \times 10^{-5}$ & 196.9 & 75.3 & $0.3174 \times 10^{-5}$ \\
\hline 15 & 7 & 1 & 11 & 290.8 & -59.4 & $0.3056 \times 10^{-5}$ & 315.8 & -47.1 & $0.2749 \times 10^{-5}$ \\
\hline 15 & 7 & 1 & 52 & 157.0 & -22.6 & $0.5928 \times 10^{-5}$ & 163.9 & -43.3 & $0.2687 \times 10^{-5}$ \\
\hline 15 & 7 & 1 & 100 & 328.8 & -64.4 & $0.7652 \times 10^{-5}$ & 31.0 & -46.4 & $0.5480 \times 10^{-5}$ \\
\hline 15 & 7 & 2 & 13 & 246.2 & -31.6 & $0.1027 \times 10^{-4}$ & 248.9 & -45.8 & $0.2688 \times 10^{-5}$ \\
\hline 15 & 7 & 2 & 50 & 209.7 & -29.1 & $0.6387 \times 10^{-5}$ & 215.9 & -48.1 & $0.3062 \times 10^{-5}$ \\
\hline 15 & 7 & 2 & 100 & 172.7 & -41.1 & - & 226.7 & -29.6 & $\longrightarrow$ \\
\hline 15 & 7 & 2 & 100 & 199.4 & 20.1 & 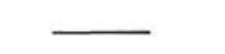 & 239.8 & 52.2 & $\longrightarrow$ \\
\hline 15 & 7 & 3 & 12 & 140.1 & 47.0 & $\longrightarrow$ & 128.6 & 69.5 & $\longrightarrow$ \\
\hline 15 & 7 & 3 & 48 & 183.9 & -11.1 & $0.2816 \times 10^{-5}$ & 246.1 & 12.1 & $0.4510 \times 10^{-6}$ \\
\hline 15 & 7 & 4 & 11 & 198.5 & -6.1 & $0.3631 \times 10^{-5}$ & 215.4 & -11.5 & $0.2199 \times 10^{-5}$ \\
\hline 15 & 7 & 4 & 52 & 282.5 & -81.6 & $0.6367 \times 10^{-5}$ & 287.2 & -70.0 & $0.4168 \times 10^{-5}$ \\
\hline 15 & 7 & 4 & 100 & 228.6 & -28.3 & $0.3917 \times 10^{-5}$ & 279.0 & -25.2 & $0.3128 \times 10^{-5}$ \\
\hline 15 & 7 & 5 & 11 & 131.8 & 2.8 & $0.4437 \times 10^{-5}$ & 85.8 & -29.1 & $0.1488 \times 10^{-5}$ \\
\hline 15 & 7 & 5 & 48 & 205.6 & -42.9 & $0.8769 \times 10^{-5}$ & 199.6 & -57.6 & $0.1695 \times 10^{-5}$ \\
\hline 15 & 7 & 5 & 100 & 206.2 & 20.2 & $0.3967 \times 10^{-5}$ & 241.3 & -6.9 & $0.2317 \times 10^{-5}$ \\
\hline 15 & 7 & 6 & 2 & 165.7 & 6.9 & $0.4983 \times 10^{-5}$ & 250.6 & 75.0 & $0.1448 \times 10^{-5}$ \\
\hline 15 & 7 & 6 & 12 & 190.2 & -55.0 & $0.8196 \times 10^{-5}$ & 252.1 & -58.5 & $0.2823 \times 10^{-5}$ \\
\hline 15 & 7 & 6 & 54 & 161.0 & 0.8 & $0.5677 \times 10^{-5}$ & 95.7 & 59.8 & $0.1712 \times 10^{-5}$ \\
\hline 15 & 7 & 6 & 102 & 206.4 & 58.5 & $0.5355 \times 10^{-5}$ & 257.0 & 55.9 & $0.3972 \times 10^{-5}$ \\
\hline 15 & 8 & 2 & 50 & 153.7 & 5.1 & $0.3590 \times 10^{-5}$ & 129.6 & -7.6 & $0.1407 \times 10^{-5}$ \\
\hline
\end{tabular}


TABLE 1-Continued

\begin{tabular}{|c|c|c|c|c|c|c|c|c|c|}
\hline \multirow[b]{2}{*}{ Hole } & \multirow[b]{2}{*}{ Core } & \multirow[b]{2}{*}{ Section } & \multirow[b]{2}{*}{$\begin{array}{l}\text { Sampled at } \\
\text { (cm) }\end{array}$} & \multicolumn{3}{|c|}{ NRM } & \multicolumn{3}{|c|}{50 oersted } \\
\hline & & & & Decl. & Incl. & $\begin{array}{l}\text { Intensity } \\
\text { emu/gm }\end{array}$ & Decl. & Incl. & $\begin{array}{l}\text { Intensity } \\
\text { emu/gm }\end{array}$ \\
\hline 15 & 8 & 3 & 2 & 266.3 & 1.1 & $0.3253 \times 10^{-4}$ & 84.8 & -68.4 & $0.3618 \times 10^{-6}$ \\
\hline 15 & 8 & 3 & 13 & 157.5 & -19.1 & $0.1978 \times 10^{-5}$ & 97.1 & -44.7 & $0.4560 \times 10^{-6}$ \\
\hline 15 & 8 & 3 & 100 & 164.9 & -71.8 & $0.5178 \times 10^{-5}$ & 186.1 & -27.0 & $0.4282 \times 10^{-6}$ \\
\hline 15 & 8 & 4 & 2 & 210.0 & -1.3 & $0.3195 \times 10^{-5}$ & 299.0 & -30.2 & $0.1733 \times 10^{-6}$ \\
\hline 15 & 8 & 4 & 12 & 152.2 & -4.3 & $0.1156 \times 10^{-5}$ & 335.5 & -61.2 & $0.2752 \times 10^{-6}$ \\
\hline 15 & 8 & 4 & 48 & 147.3 & -7.7 & $0.3507 \times 10^{-5}$ & 66.1 & -48.1 & $0.6748 \times 10^{-6}$ \\
\hline 15 & 8 & 4 & 103 & 182.5 & -23.7 & $0.1916 \times 10^{-5}$ & 170.5 & -30.6 & $0.3895 \times 10^{-6}$ \\
\hline 15 & 8 & 5 & 2 & 132.9 & -46.7 & $0.3284 \times 10^{-5}$ & 225.9 & -39.0 & $0.4154 \times 10^{-6}$ \\
\hline 15 & 8 & 5 & 11 & 128.6 & -14.9 & $0.2100 \times 10^{-5}$ & 58.8 & -29.9 & $0.7076 \times 10^{-6}$ \\
\hline 15 & 8 & 5 & 50 & 222.0 & -4.7 & $\longrightarrow$ & 214.8 & -22.4 & $\longrightarrow$ \\
\hline 15 & 8 & 5 & 100 & 161.6 & -24.9 & $0.1849 \times 10^{-5}$ & 217.3 & -20.5 & $0.4781 \times 10^{-6}$ \\
\hline 15 & 8 & 6 & 13 & 160.7 & -21.4 & $\longrightarrow$ & 169.8 & -8.3 & $\longrightarrow$ \\
\hline 15 & 8 & 6 & 51 & 139.3 & -7.8 & $0.4062 \times 10^{-5}$ & 246.6 & -30.3 & $0.9087 \times 10^{-6}$ \\
\hline 15 & 9 & 1 & 48 & 175.6 & 20.5 & $0.2608 \times 10^{-5}$ & 172.8 & 20.2 & $0.8032 \times 10^{-6}$ \\
\hline 15 & 9 & 1 & 110 & 226.0 & -48.5 & $0.6513 \times 10^{-4}$ & 237.5 & -46.4 & $0.6214 \times 10^{-4}$ \\
\hline 15 & 9 & 2 & 52 & 166.3 & -17.4 & $0.5339 \times 10^{-5}$ & 150.4 & 2.0 & $0.1330 \times 10^{-5}$ \\
\hline 15 & 9 & 2 & 105 & 201.8 & 8.5 & $0.4102 \times 10^{-4}$ & 201.6 & 5.7 & $0.3490 \times 10^{-4}$ \\
\hline 15 & 9 & 3 & 2 & 142.4 & 13.9 & $0.4945 \times 10^{-5}$ & 104.0 & 49.1 & $0.1290 \times 10^{-5}$ \\
\hline 15 & 9 & 3 & 51 & 203.1 & -3.8 & $0.4534 \times 10^{-5}$ & 196.5 & -18.3 & $0.1122 \times 10^{-5}$ \\
\hline 15 & 9 & 3 & 104 & 171.0 & -16.3 & $0.1698 \times 10^{-5}$ & 299.7 & -53.7 & $0.7320 \times 10^{-6}$ \\
\hline 15 & 9 & 4 & 52 & 160.7 & 19.7 & $0.6894 \times 10^{-5}$ & 149.1 & 46.7 & $0.3267 \times 10^{-5}$ \\
\hline 15 & 9 & 4 & 100 & 123.3 & 59.1 & $0.7020 \times 10^{-5}$ & 25.2 & 60.5 & $0.4842 \times 10^{-5}$ \\
\hline 15 & 9 & 5 & 2 & 226.8 & 35.4 & $0.6258 \times 10^{-5}$ & 250.4 & 37.4 & $0.3061 \times 10^{-5}$ \\
\hline 15 & 9 & 5 & 11 & 203.2 & -43.2 & $0.6214 \times 10^{-5}$ & 222.9 & 49.1 & $0.4346 \times 10^{-5}$ \\
\hline 15 & 9 & 5 & 53 & 141.4 & -49.5 & $0.3455 \times 10^{-5}$ & 100.8 & -70.6 & $0.2745 \times 10^{-5}$ \\
\hline 15 & 9 & 5 & 100 & 195.9 & 24.4 & $0.3026 \times 10^{-5}$ & 237.6 & -46.4 & $0.1861 \times 10^{-5}$ \\
\hline 15 & 9 & 6 & 10 & 116.6 & -38.6 & $0.2890 \times 10^{-5}$ & 70.4 & -54.5 & $0.2102 \times 10^{-5}$ \\
\hline 15 & 9 & 6 & 51 & 178.7 & -27.6 & $\longrightarrow$ & 174.9 & -38.8 & $\longrightarrow$ \\
\hline 16 & 1 & 1 & 12 & 357.4 & 2.8 & $0.4924 \times 10^{-5}$ & 341.9 & 9.3 & $0.2067 \times 10^{-5}$ \\
\hline
\end{tabular}


TABLE 1-Continued

\begin{tabular}{|c|c|c|c|c|c|c|c|c|c|}
\hline \multirow[b]{2}{*}{ Hole } & \multirow[b]{2}{*}{ Core } & \multirow[b]{2}{*}{ Section } & \multirow[b]{2}{*}{$\begin{array}{l}\text { Sampled at } \\
(\mathrm{cm})\end{array}$} & \multicolumn{3}{|c|}{ NRM } & \multicolumn{3}{|c|}{50 oersted } \\
\hline & & & & Decl. & Incl. & $\begin{array}{l}\text { Intensity } \\
\text { emu/gm }\end{array}$ & Decl. & Incl. & $\begin{array}{l}\text { Intensity } \\
\text { emu/gm }\end{array}$ \\
\hline 16 & 1 & 1 & 48 & 245.8 & 11.2 & $0.7603 \times 10^{-5}$ & 268.3 & 6.2 & $0.6597 \times 10^{-5}$ \\
\hline 16 & 1 & 1 & 100 & 140.5 & 7.7 & $0.1004 \times 10^{-5}$ & 51.4 & -23.1 & $0.6909 \times 10^{-6}$ \\
\hline 16 & 1 & 2 & 100 & 0.1 & -2.6 & $\longrightarrow$ & 348.6 & -42.1 & $\longrightarrow$ \\
\hline 16 & 2 & 6 & 2 & 171.7 & 29.8 & $0.2662 \times 10^{-5}$ & 167.0 & 38.4 & $0.1209 \times 10^{-5}$ \\
\hline 16 & 2 & 6 & 11 & 122.7 & 17.5 & $0.1233 \times 10^{-5}$ & 94.5 & -33.0 & $0.6081 \times 10^{-6}$ \\
\hline$* 16$ & 2 & 6 & $50-2$ & 211.7 & -21.2 & $0.1579 \times 10^{-5}$ & 228.8 & 43.2 & $0.5712 \times 10^{-6}$ \\
\hline 16 & 4 & 1 & 52 & 198.7 & 4.7 & $0.3380 \times 10^{-5}$ & 219.4 & 15.6 & $0.1781 \times 10^{-5}$ \\
\hline 16 & 4 & 1 & 100 & 153.7 & 0.6 & $0.2270 \times 10^{-5}$ & 147.4 & -15.4 & $0.7613 \times 10^{-6}$ \\
\hline 16 & 4 & 2 & 11 & 172.4 & 0.3 & $0.2204 \times 10^{-5}$ & 173.3 & -18.9 & $0.1470 \times 10^{-5}$ \\
\hline 16 & 4 & 2 & 100 & 134.2 & 8.3 & $0.1035 \times 10^{-5}$ & 190.4 & 21.8 & $0.2976 \times 10^{-6}$ \\
\hline 16 & 4 & 3 & 13 & 194.7 & 35.4 & $\longrightarrow$ & 216.0 & 32.2 & $\longrightarrow$ \\
\hline 16 & 4 & 3 & 54 & 168.4 & 27.2 & $0.2079 \times 10^{-5}$ & 159.0 & 41.7 & $0.1278 \times 10^{-5}$ \\
\hline 16 & 4 & 3 & 100 & 163.5 & -0.8 & $0.1327 \times 10^{-5}$ & 185.5 & 12.6 & $0.3080 \times 10^{-6}$ \\
\hline 16 & 4 & 4 & 10 & 165.4 & 29.4 & $0.8978 \times 10^{-6}$ & 152.1 & 41.4 & $0.5042 \times 10^{-6}$ \\
\hline 16 & 4 & 4 & 50 & 175.6 & -11.7 & $0.2217 \times 10^{-5}$ & 201.1 & -3.7 & $0.1398 \times 10^{-5}$ \\
\hline 16 & 4 & 4 & 100 & 171.6 & 25.3 & $0.1378 \times 10^{-5}$ & 175.4 & 19.7 & $0.8010 \times 10^{-6}$ \\
\hline 16 & 4 & 5 & 12 & 160.7 & -3.2 & $0.1008 \times 10^{-5}$ & 163.3 & 10.8 & $0.5053 \times 10^{-6}$ \\
\hline 16 & 4 & 5 & 50 & 178.4 & -23.9 & $0.2510 \times 10^{-5}$ & 170.4 & -32.0 & $0.1202 \times 10^{-5}$ \\
\hline 16 & 4 & 5 & 100 & 183.1 & -4.7 & $0.1455 \times 10^{-5}$ & 177.7 & -12.1 & $0.7156 \times 10^{-6}$ \\
\hline 17 & 1 & 1 & 53 & 175.6 & 22.0 & $0.3938 \times 10^{-5}$ & 186.1 & 36.3 & $0.1232 \times 10^{-5}$ \\
\hline 17 & 1 & 2 & 50 & 197.9 & -14.8 & $0.4197 \times 10^{-5}$ & 183.4 & -11.7 & $0.1504 \times 10^{-5}$ \\
\hline 17 & 1 & 4 & 50 & 182.0 & 3.7 & $0.2552 \times 10^{-5}$ & 92.1 & -1.9 & $0.1245 \times 10^{-4}$ \\
\hline 17 & 1 & 5 & 50 & 156.4 & -4.6 & $0.3576 \times 10^{-5}$ & 133.8 & -8.7 & $0.8343 \times 10^{-6}$ \\
\hline 17 & 2 & 1 & 23 & 180.9 & 28.5 & $0.1361 \times 10^{-5}$ & 172.4 & 56.2 & $0.9210 \times 10^{-6}$ \\
\hline 17 & 2 & 1 & 52 & 236.7 & 64.2 & $0.1565 \times 10^{-3}$ & 240.9 & 63.1 & $0.1451 \times 10^{-3}$ \\
\hline 17 & 2 & 2 & 50 & 231.1 & 49.4 & $0.1667 \times 10^{-4}$ & 235.8 & 35.9 & $0.1159 \times 10^{-4}$ \\
\hline 17 & 2 & 2 & 100 & 175.4 & 11.2 & $0.8570 \times 10^{-6}$ & 174.4 & 18.7 & $0.3644 \times 10^{-6}$ \\
\hline 17 & 2 & 3 & 13 & 164.6 & -20.7 & $0.1451 \times 10^{-5}$ & 152.5 & -38.7 & $0.6142 \times 10^{-6}$ \\
\hline$* 17$ & 2 & 3 & $54-6$ & 147.9 & -4.9 & $0.5882 \times 10^{-6}$ & 154.9 & -37.8 & $0.2024 \times 10^{-6}$ \\
\hline
\end{tabular}


TABLE 1-Continued

\begin{tabular}{|c|c|c|c|c|c|c|c|c|c|}
\hline \multirow[b]{2}{*}{ Hole } & \multirow[b]{2}{*}{ Core } & \multirow[b]{2}{*}{ Section } & \multirow[b]{2}{*}{$\begin{array}{l}\text { Sampled at } \\
(\mathrm{cm})\end{array}$} & \multicolumn{3}{|c|}{ NRM } & \multicolumn{3}{|c|}{50 oersted } \\
\hline & & & & Decl. & Incl. & $\begin{array}{l}\text { Intensity } \\
\text { emu/gm }\end{array}$ & Decl. & Incl. & $\begin{array}{l}\text { Intensity } \\
\text { emu/gm }\end{array}$ \\
\hline 17 & 2 & 3 & 99 & 160.5 & -20.5 & $0.1114 \times 10^{-5}$ & 157.3 & -30.5 & $0.3941 \times 10^{-6}$ \\
\hline 17 & 2 & 4 & 14 & 129.9 & -2.9 & $0.8327 \times 10^{-6}$ & 106.9 & -14.0 & $0.2134 \times 10^{-6}$ \\
\hline 17 & 2 & 4 & 50 & 158.7 & -8.0 & $0.6165 \times 10^{-6}$ & 34.2 & 26.2 & $0.2861 \times 10^{-6}$ \\
\hline 17 & 2 & 4 & 114 & 202.3 & -6.2 & $0.4343 \times 10^{-6}$ & 179.5 & -0.4 & $0.4111 \times 10^{-5}$ \\
\hline 17 & 2 & 5 & 40 & 92.8 & 38.3 & $\longrightarrow$ & 47.1 & 37.2 & $\longrightarrow$ \\
\hline 17 & 2 & 5 & 100 & 160.3 & -44.7 & $0.4084 \times 10^{-6}$ & 132.1 & -56.6 & $0.2466 \times 10^{-6}$ \\
\hline 17 & 2 & 6 & 13 & 162.4 & 3.1 & $0.2772 \times 10^{-6}$ & 126.8 & -41.7 & $0.1628 \times 10^{-6}$ \\
\hline 17 & 2 & 6 & 50 & 178.0 & -13.2 & $0.2779 \times 10^{-5}$ & 170.9 & -29.7 & $0.5452 \times 10^{-6}$ \\
\hline $17 \mathrm{~A}$ & 1 & 1 & 50 & 184.0 & 18.0 & $0.6540 \times 10^{-5}$ & 182.9 & 25.9 & $0.2547 \times 10^{-5}$ \\
\hline $17 \mathrm{~A}$ & 1 & 1 & 100 & 164.8 & 1.0 & $0.5600 \times 10^{-5}$ & 198.7 & 2.2 & $0.1752 \times 10^{-5}$ \\
\hline $17 \mathrm{~A}$ & 1 & 2 & 12 & 194.3 & -21.5 & $0.3233 \times 10^{-5}$ & 201.8 & -40.2 & $0.9652 \times 10^{-6}$ \\
\hline $17 \mathrm{~A}$ & 1 & 2 & 50 & 159.6 & 11.4 & $0.6549 \times 10^{-5}$ & 127.3 & 22.7 & $0.2334 \times 10^{-5}$ \\
\hline $17 \mathrm{~A}$ & 1 & 3 & 51 & 215.0 & -43.6 & $0.5034 \times 10^{-5}$ & 134.4 & -76.5 & $0.3095 \times 10^{-5}$ \\
\hline $17 \mathrm{~A}$ & 1 & 4 & 50 & 137.6 & -43.4 & $0.1147 \times 10^{-4}$ & 111.7 & 13.8 & $0.2156 \times 10^{-5}$ \\
\hline $17 \mathrm{~A}$ & 1 & 4 & 100 & 189.5 & -4.8 & $0.5328 \times 10^{-5}$ & 88.6 & -4.1 & $0.1180 \times 10^{-5}$ \\
\hline $17 \mathrm{~A}$ & 1 & 5 & 7 & 192.5 & -30.1 & $0.6126 \times 10^{-5}$ & 13.6 & -43.1 & $0.1133 \times 10^{-5}$ \\
\hline $17 \mathrm{~A}$ & 1 & 5 & 50 & 138.5 & 36.4 & $0.1150 \times 10^{-4}$ & 77.4 & -24.6 & $0.1833 \times 10^{-5}$ \\
\hline $17 \mathrm{~A}$ & 1 & 5 & 100 & 186.1 & 16.2 & $0.4999 \times 10^{-5}$ & 131.1 & 63.3 & $0.7969 \times 10^{-6}$ \\
\hline $17 \mathrm{~A}$ & 1 & 6 & 17 & 204.5 & -10.1 & $0.4257 \times 10^{-5}$ & 213.6 & -30.3 & $0.1435 \times 10^{-5}$ \\
\hline $17 \mathrm{~A}$ & 2 & 1 & 69 & 177.4 & -1.9 & $0.8709 \times 10^{-5}$ & 120.4 & -13.0 & $0.1778 \times 10^{-5}$ \\
\hline $17 \mathrm{~A}$ & 2 & 2 & 1 & 186.5 & -43.2 & $0.2578 \times 10^{-5}$ & 273.9 & -78.4 & $0.2696 \times 10^{-5}$ \\
\hline $17 \mathrm{~A}$ & 2 & 2 & 100 & 184.8 & -5.7 & $\longrightarrow$ & 201.1 & -31.1 & $\longrightarrow$ \\
\hline $17 \mathrm{~A}$ & 2 & 3 & 12 & 184.6 & -13.3 & $\longrightarrow$ & 246.5 & -55.1 & - \\
\hline $17 \mathrm{~A}$ & 2 & 4 & 12 & 191.0 & 4.6 & $0.3676 \times 10^{-5}$ & 205.2 & -24.8 & $0.9814 \times 10^{-6}$ \\
\hline$* 17 \mathrm{~A}$ & 2 & 4 & $50-2$ & 189.5 & -17.7 & $0.4022 \times 10^{-5}$ & 196.2 & -34.8 & $0.1065 \times 10^{-5}$ \\
\hline $17 \mathrm{~A}$ & 2 & 4 & 100 & 234.2 & 31.8 & $0.3580 \times 10^{-5}$ & 301.7 & 58.7 & $0.2548 \times 10^{-5}$ \\
\hline $17 \mathrm{~A}$ & 2 & 5 & 2 & 174.5 & 31.7 & $0.3786 \times 10^{-5}$ & 153.2 & 50.2 & $0.1806 \times 10^{-5}$ \\
\hline $17 \mathrm{~A}$ & 2 & 5 & 62 & 182.8 & -10.7 & $0.4996 \times 10^{-5}$ & 220.9 & -37.3 & $0.1572 \times 10^{-5}$ \\
\hline
\end{tabular}


TABLE 1-Continued

\begin{tabular}{|c|c|c|c|c|c|c|c|c|c|}
\hline \multirow[b]{2}{*}{ Hole } & \multirow[b]{2}{*}{ Core } & \multirow[b]{2}{*}{ Section } & \multirow[b]{2}{*}{$\begin{array}{l}\text { Sampled at } \\
\text { (cm) }\end{array}$} & \multicolumn{3}{|c|}{ NRM } & \multicolumn{3}{|c|}{50 oersted } \\
\hline & & & & Decl. & Incl. & $\begin{array}{l}\text { Intensity } \\
\text { emu/gm }\end{array}$ & Decl. & Incl. & $\begin{array}{l}\text { Intensity } \\
\text { emu/gm }\end{array}$ \\
\hline $17 \mathrm{~A}$ & 2 & 5 & 100 & 181.0 & 18.8 & $0.1580 \times 10^{-5}$ & 124.1 & 53.0 & $0.7653 \times 10^{-6}$ \\
\hline $17 \mathrm{~A}$ & 2 & 6 & 19 & 238.5 & -28.8 & $0.2117 \times 10^{-5}$ & 257.7 & -37.7 & $0.9664 \times 10^{-6}$ \\
\hline $17 \mathrm{~A}$ & 2 & 6 & 50 & 174.3 & -7.8 & $0.4682 \times 10^{-5}$ & 169.3 & -19.9 & $0.1210 \times 10^{-5}$ \\
\hline $17 \mathrm{~A}$ & 2 & 6 & 100 & 181.5 & -12.9 & $0.1838 \times 10^{-5}$ & 157.7 & -61.6 & $0.3445 \times 10^{-6}$ \\
\hline $17 \mathrm{~A}$ & 3 & 1 & 53 & 207.3 & 19.4 & $0.1862 \times 10^{-5}$ & 220.7 & 26.3 & $0.8543 \times 10^{-6}$ \\
\hline $17 \mathrm{~A}$ & 3 & 1 & 100 & 162.8 & 19.9 & $0.1734 \times 10^{-5}$ & 161.8 & 15.8 & $0.7381 \times 10^{-6}$ \\
\hline $17 \mathrm{~A}$ & 4 & 2 & 8 & 182.6 & 0.8 & $0.1088 \times 10^{-5}$ & 146.3 & -63.8 & $0.2035 \times 10^{-6}$ \\
\hline $17 \mathrm{~A}$ & 4 & 2 & 50 & 196.6 & -37.6 & $0.3516 \times 10^{-5}$ & 240.1 & -79.9 & $0.1624 \times 10^{-5}$ \\
\hline $17 \mathrm{~A}$ & 4 & 2 & 75 & 117.3 & -55.8 & $0.6680 \times 10^{-5}$ & 63.3 & -65.0 & $0.4908 \times 10^{-5}$ \\
\hline $17 \mathrm{~A}$ & 4 & 2 & 100 & 169.1 & -47.9 & $0.4544 \times 10^{-5}$ & 156.1 & -72.5 & $0.2652 \times 10^{-5}$ \\
\hline $17 \mathrm{~A}$ & 4 & 6 & 6 & 196.0 & 17.9 & $0.9800 \times 10^{-6}$ & 197.7 & 50.6 & $0.3041 \times 10^{-6}$ \\
\hline $17 \mathrm{~A}$ & 4 & 6 & 50 & 182.8 & -4.9 & $0.2773 \times 10^{-5}$ & 201.5 & -27.0 & $0.5408 \times 10^{-6}$ \\
\hline $17 \mathrm{~A}$ & 4 & 6 & 75 & 199.3 & -22.5 & $0.4828 \times 10^{-5}$ & 176.3 & -50.6 & $0.2191 \times 10^{-5}$ \\
\hline $17 \mathrm{~A}$ & 4 & 6 & 105 & 51.0 & 31.6 & $0.4195 \times 10^{-6}$ & 43.8 & 13.2 & $0.6051 \times 10^{-6}$ \\
\hline $17 \mathrm{~B}$ & 1 & 1 & 16 & 124.9 & -5.5 & $0.1568 \times 10^{-5}$ & 62.9 & 5.7 & $0.1129 \times 10^{-5}$ \\
\hline $17 \mathrm{~B}$ & 1 & 1 & 50 & 195.9 & 2.2 & $0.5421 \times 10^{-5}$ & 220.0 & 16.2 & $0.1761 \times 10^{-5}$ \\
\hline $17 \mathrm{~B}$ & 1 & 1 & 100 & 168.8 & -33.1 & $0.2557 \times 10^{-5}$ & 199.1 & -25.9 & $0.7112 \times 10^{-6}$ \\
\hline $17 \mathrm{~B}$ & 1 & 2 & 2 & 203.8 & -31.1 & $0.3741 \times 10^{-5}$ & 203.6 & -39.8 & $0.1417 \times 10^{-5}$ \\
\hline $17 \mathrm{~B}$ & 1 & 2 & 50 & 213.8 & -12.2 & $0.4359 \times 10^{-5}$ & 263.9 & -33.7 & $0.2016 \times 10^{-5}$ \\
\hline $17 \mathrm{~B}$ & 1 & 2 & 100 & 174.7 & -33.9 & $0.4522 \times 10^{-5}$ & 142.1 & -37.3 & $0.3462 \times 10^{-5}$ \\
\hline $17 \mathrm{~B}$ & 1 & 3 & 7 & 183.6 & -20.9 & $0.3294 \times 10^{-5}$ & 159.3 & -23.6 & $0.1905 \times 10^{-5}$ \\
\hline $17 \mathrm{~B}$ & 1 & 3 & 50 & 174.7 & -1.7 & $0.3451 \times 10^{-5}$ & 140.0 & -38.9 & $0.7455 \times 10^{-6}$ \\
\hline $17 \mathrm{~B}$ & 1 & 3 & 100 & 105.3 & 57.7 & $0.9312 \times 10^{-6}$ & 176.1 & -9.0 & $0.1785 \times 10^{-6}$ \\
\hline $17 \mathrm{~B}$ & 1 & 4 & 16 & 205.2 & -0.4 & $0.1302 \times 10^{-5}$ & 69.4 & -11.2 & $0.3186 \times 10^{-6}$ \\
\hline $17 \mathrm{~B}$ & 1 & 4 & 50 & 194.7 & 10.3 & $0.3463 \times 10^{-5}$ & 247.5 & 32.9 & $0.8705 \times 10^{-6}$ \\
\hline $17 \mathrm{~B}$ & 1 & 4 & 100 & 212.7 & 6.8 & $0.1832 \times 10^{-5}$ & 238.9 & 20.3 & $0.7531 \times 10^{-6}$ \\
\hline $17 \mathrm{~B}$ & 1 & 5 & 32 & 198.9 & 11.2 & $0.2128 \times 10^{-5}$ & 230.6 & -15.2 & $0.5601 \times 10^{-6}$ \\
\hline $17 \mathrm{~B}$ & 1 & 5 & 50 & 202.4 & -7.6 & $0.5010 \times 10^{-5}$ & 220.1 & -12.7 & $0.2045 \times 10^{-5}$ \\
\hline 17B & 1 & 5 & 100 & 243.4 & -26.9 & $0.3211 \times 10^{-5}$ & 269.1 & -24.8 & $0.2059 \times 10^{-5}$ \\
\hline
\end{tabular}


TABLE 1-Continued

\begin{tabular}{|c|c|c|c|c|c|c|c|c|c|}
\hline \multirow[b]{2}{*}{ Hole } & \multirow[b]{2}{*}{ Core } & \multirow[b]{2}{*}{ Section } & \multirow[b]{2}{*}{$\begin{array}{l}\text { Sampled at } \\
\text { (cm) }\end{array}$} & \multicolumn{3}{|c|}{ NRM } & \multicolumn{3}{|c|}{50 oersted } \\
\hline & & & & Decl. & Incl. & $\begin{array}{l}\text { Intensity } \\
\text { emu/gm }\end{array}$ & Decl. & Incl. & $\begin{array}{l}\text { Intensity } \\
\text { emu/gm }\end{array}$ \\
\hline 17B & 1 & 6 & 50 & 191.3 & -17.8 & $\longrightarrow$ & 206.5 & -26.3 & $\longrightarrow$ \\
\hline 17B & 1 & 6 & 100 & 218.2 & -4.3 & $0.1416 \times 10^{-5}$ & 321.1 & 45.2 & $0.5349 \times 10^{-6}$ \\
\hline $17 \mathrm{~B}$ & 2 & 1 & 130 & 248.0 & 46.3 & $0.4567 \times 10^{-5}$ & 311.3 & 50.2 & $0.2880 \times 10^{-5}$ \\
\hline $17 \mathrm{~B}$ & 2 & 2 & 14 & 274.0 & 76.6 & $0.2376 \times 10^{-5}$ & 311.4 & 49.6 & $0.2468 \times 10^{-5}$ \\
\hline $17 \mathrm{~B}$ & 2 & 2 & 50 & 217.6 & 10.1 & $0.3496 \times 10^{-5}$ & 71.5 & 4.5 & $0.2068 \times 10^{-5}$ \\
\hline $17 \mathrm{~B}$ & 2 & 2 & 100 & 171.3 & 14.8 & $0.3801 \times 10^{-5}$ & 147.5 & 11.6 & $0.2014 \times 10^{-5}$ \\
\hline 17B & 2 & 3 & 7 & 213.2 & 18.1 & $0.2584 \times 10^{-5}$ & 257.6 & 21.7 & $0.5978 \times 10^{-6}$ \\
\hline $17 \mathrm{~B}$ & 2 & 3 & 50 & 171.8 & 18.7 & $0.5726 \times 10^{-5}$ & 176.0 & 25.9 & $0.1618 \times 10^{-5}$ \\
\hline $17 \mathrm{~B}$ & 2 & 3 & 100 & 16.9 & -16.2 & $0.2627 \times 10^{-5}$ & 190.7 & -41.2 & $0.3786 \times 10^{-6}$ \\
\hline 17B & 2 & 4 & 16 & 183.5 & 28.6 & $0.2232 \times 10^{-5}$ & 95.3 & 33.7 & $0.5213 \times 10^{-6}$ \\
\hline $17 \mathrm{~B}$ & 2 & 4 & 50 & 189.0 & 82.2 & $0.1882 \times 10^{-4}$ & 57.0 & 76.0 & $0.1423 \times 10^{-5}$ \\
\hline 17B & 2 & 4 & 101 & 164.2 & 19.3 & $0.1899 \times 10^{-5}$ & 79.6 & 47.4 & $0.5099 \times 10^{-6}$ \\
\hline 17B & 2 & 5 & 15 & 205.0 & 35.0 & $0.3110 \times 10^{-5}$ & 277.1 & 58.9 & $0.5819 \times 10^{-6}$ \\
\hline $17 \mathrm{~B}$ & 2 & 5 & 50 & 162.9 & 14.0 & $0.4315 \times 10^{-5}$ & 133.5 & 23.3 & $0.1790 \times 10^{-5}$ \\
\hline 17B & 2 & 5 & 100 & 181.9 & -1.9 & $0.2053 \times 10^{-5}$ & 191.5 & -31.0 & $0.3972 \times 10^{-6}$ \\
\hline 17B & 2 & 6 & 7 & 205.7 & -1.5 & $0.2737 \times 10^{-5}$ & 241.1 & 32.2 & $0.8526 \times 10^{-6}$ \\
\hline $17 \mathrm{~B}$ & 2 & 6 & 66 & 178.1 & -9.8 & $0.5694 \times 10^{-5}$ & 175.9 & -29.3 & $0.2539 \times 10^{-5}$ \\
\hline 17B & 2 & 6 & 100 & 210.6 & 10.8 & $0.2126 \times 10^{-5}$ & 280.7 & 13.1 & $0.2921 \times 10^{-6}$ \\
\hline 17B & 3 & 1 & 17 & 177.8 & 63.3 & $0.7265 \times 10^{-5}$ & 195.3 & 77.0 & $0.5393 \times 10^{-5}$ \\
\hline 17B & 3 & 1 & 48 & 187.9 & 5.4 & $0.5659 \times 10^{-5}$ & 193.2 & 2.5 & $0.1327 \times 10^{-5}$ \\
\hline 17B & 3 & 1 & 100 & 148.1 & 40.3 & $0.4993 \times 10^{-5}$ & 105.7 & -52.3 & $0.3539 \times 10^{-5}$ \\
\hline $17 \mathrm{~B}$ & 3 & 2 & 15 & 198.4 & -15.1 & $0.1621 \times 10^{-5}$ & 200.1 & -30.7 & $0.5256 \times 10^{-6}$ \\
\hline $17 \mathrm{~B}$ & 3 & 2 & 50 & 213.4 & 7.6 & $0.3167 \times 10^{-5}$ & 259.5 & -14.0 & $0.9437 \times 10^{-6}$ \\
\hline 17B & 3 & 2 & 100 & 209.5 & -3.6 & $0.2330 \times 10^{-5}$ & 232.0 & -6.5 & $0.1237 \times 10^{-5}$ \\
\hline 17B & 3 & 3 & 17 & 286.4 & -27.8 & $0.3736 \times 10^{-5}$ & 314.5 & -36.8 & $0.3341 \times 10^{-5}$ \\
\hline $17 \mathrm{~B}$ & 3 & 3 & 50 & 208.7 & -20.5 & $0.6050 \times 10^{-5}$ & 236.7 & -33.6 & $0.3152 \times 10^{-5}$ \\
\hline 17B & 3 & 3 & 100 & 196.6 & 30.7 & $0.2576 \times 10^{-5}$ & 247.5 & -30.1 & $0.8672 \times 10^{-6}$ \\
\hline 17B & 3 & 4 & 16 & 200.4 & -29.2 & $0.2337 \times 10^{-5}$ & 188.1 & -77.3 & $0.9181 \times 10^{-6}$ \\
\hline $17 \mathrm{~B}$ & 3 & 4 & 50 & 178.0 & 1.7 & $0.2398 \times 10^{-5}$ & 209.9 & -14.7 & $0.8352 \times 10^{-6}$ \\
\hline
\end{tabular}


TABLE 1-Continued

\begin{tabular}{|c|c|c|c|c|c|c|c|c|c|}
\hline \multirow[b]{2}{*}{ Hole } & \multirow[b]{2}{*}{ Core } & \multirow[b]{2}{*}{ Section } & \multirow[b]{2}{*}{$\begin{array}{l}\text { Sampled at } \\
(\mathrm{cm})\end{array}$} & \multicolumn{3}{|c|}{ NRM } & \multicolumn{3}{|c|}{50 oersted } \\
\hline & & & & Decl. & Incl. & $\begin{array}{l}\text { Intensity } \\
\text { emu/gm }\end{array}$ & Decl. & Incl. & $\begin{array}{l}\text { Intensity } \\
\text { emu/gm }\end{array}$ \\
\hline $17 \mathrm{~B}$ & 3 & 4 & 100 & 194.8 & 11.8 & $0.3238 \times 10^{-5}$ & 202.6 & -21.0 & $0.1493 \times 10^{-5}$ \\
\hline $17 \mathrm{~B}$ & 3 & 5 & 27 & 205.2 & -32.7 & $0.6053 \times 10^{-5}$ & 219.5 & -20.3 & $0.7549 \times 10^{-6}$ \\
\hline $17 \mathrm{~B}$ & 3 & 5 & 101 & 201.3 & 13.3 & $0.3590 \times 10^{-5}$ & 199.6 & 2.8 & $0.1024 \times 10^{-5}$ \\
\hline 17B & 3 & 6 & 25 & 154.1 & -22.2 & $0.5830 \times 10^{-5}$ & 152.9 & -25.2 & $0.4475 \times 10^{-5}$ \\
\hline $17 \mathrm{~B}$ & 3 & 6 & 46 & 232.6 & -39.3 & $0.4687 \times 10^{-5}$ & 268.1 & -44.2 & $0.3178 \times 10^{-5}$ \\
\hline $17 \mathrm{~B}$ & 3 & 6 & 100 & 198.8 & -40.3 & $0.1907 \times 10^{-5}$ & 198.3 & -73.9 & $0.9210 \times 10^{-6}$ \\
\hline $17 \mathrm{~B}$ & 4 & 1 & 34 & 239.7 & -47.1 & $0.6223 \times 10^{-5}$ & 252.2 & -48.7 & $0.4452 \times 10^{-5}$ \\
\hline $17 \mathrm{~B}$ & 4 & 1 & 50 & 224.3 & -20.9 & $0.8302 \times 10^{-5}$ & 239.2 & -22.9 & $0.4970 \times 10^{-5}$ \\
\hline $17 \mathrm{~B}$ & 4 & 1 & 100 & 229.5 & -13.6 & $0.2864 \times 10^{-5}$ & 244.6 & -25.9 & $0.1593 \times 10^{-5}$ \\
\hline $17 \mathrm{~B}$ & 4 & 2 & 6 & 220.5 & -6.4 & $0.4860 \times 10^{-5}$ & 233.1 & -15.5 & $0.3312 \times 10^{-5}$ \\
\hline $17 \mathrm{~B}$ & 4 & 2 & 50 & 222.8 & -12.7 & $0.5691 \times 10^{-5}$ & 250.5 & -20.1 & $0.3014 \times 10^{-5}$ \\
\hline $17 \mathrm{~B}$ & 4 & 2 & 102 & 183.0 & 17.5 & $0.1033 \times 10^{-5}$ & 46.2 & -43.3 & $0.2578 \times 10^{-6}$ \\
\hline $17 \mathrm{~B}$ & 4 & 3 & 2 & 64.9 & -20.2 & $0.2301 \times 10^{-5}$ & 45.5 & -26.8 & $0.2818 \times 10^{-5}$ \\
\hline $17 \mathrm{~B}$ & 4 & 3 & 51 & 173.3 & -18.8 & $0.6303 \times 10^{-5}$ & 167.4 & -49.5 & $0.2287 \times 10^{-5}$ \\
\hline $17 \mathrm{~B}$ & 4 & 3 & 100 & 172.5 & 23.6 & $0.9821 \times 10^{-6}$ & 55.7 & 3.0 & $0.3681 \times 10^{-6}$ \\
\hline $17 \mathrm{~B}$ & 4 & 4 & 15 & 155.6 & 15.7 & $0.2889 \times 10^{-5}$ & 127.0 & 7.0 & $0.1998 \times 10^{-5}$ \\
\hline 17B & 4 & 4 & 50 & 175.3 & -13.3 & $0.3543 \times 10^{-5}$ & 170.6 & -32.9 & $0.1309 \times 10^{-5}$ \\
\hline $17 \mathrm{~B}$ & 4 & 4 & 102 & 255.2 & 31.3 & $0.1871 \times 10^{-5}$ & 311.8 & 28.1 & $0.1029 \times 10^{-5}$ \\
\hline $17 \mathrm{~B}$ & 4 & 5 & 4 & 201.4 & 7.4 & $0.1721 \times 10^{-5}$ & 204.8 & 11.5 & $0.5866 \times 10^{-6}$ \\
\hline $17 \mathrm{~B}$ & 4 & 5 & 50 & 187.6 & -5.8 & $0.4655 \times 10^{-5}$ & 188.7 & -5.6 & $0.1858 \times 10^{-5}$ \\
\hline $17 \mathrm{~B}$ & 4 & 5 & 100 & 245.0 & 7.6 & $0.2271 \times 10^{-5}$ & 268.6 & 7.8 & $0.1573 \times 10^{-5}$ \\
\hline $17 \mathrm{~B}$ & 4 & 6 & 3 & 172.9 & -14.6 & $0.1986 \times 10^{-5}$ & 94.6 & -58.7 & $0.7147 \times 10^{-6}$ \\
\hline$* 17 \mathrm{~B}$ & 4 & 6 & $53-5$ & 119.0 & 37.3 & $0.3782 \times 10^{-5}$ & 88.9 & 30.9 & $0.2551 \times 10^{-5}$ \\
\hline $17 \mathrm{~B}$ & 4 & 6 & 101 & 208.7 & -35.2 & $0.3118 \times 10^{-5}$ & 255.1 & -6.3 & $0.1297 \times 10^{-5}$ \\
\hline 18 & 2 & 2 & 3 & 42.7 & -67.3 & $0.6812 \times 10^{-5}$ & 50.5 & -58.6 & $0.5715 \times 10^{-5}$ \\
\hline 18 & 2 & 2 & 100 & 276.9 & -65.8 & $0.4287 \times 10^{-5}$ & 352.3 & -71.9 & $0.3785 \times 10^{-5}$ \\
\hline 18 & 3 & 2 & 2 & 341.8 & -69.1 & $0.2644 \times 10^{-5}$ & 191.1 & -59.5 & $0.2323 \times 10^{-5}$ \\
\hline 18 & 3 & 2 & 103 & 63.4 & -88.9 & $0.5220 \times 10^{-5}$ & - & $\longrightarrow$ & - \\
\hline 18 & 4 & 4 & 6 & 121.8 & -62.4 & $0.2995 \times 10^{-5}$ & 104.7 & -52.8 & $0.1999 \times 10^{-5}$ \\
\hline
\end{tabular}


TABLE 1-Continued

\begin{tabular}{|c|c|c|c|c|c|c|c|c|c|}
\hline \multirow[b]{2}{*}{ Hole } & \multirow[b]{2}{*}{ Core } & \multirow[b]{2}{*}{ Section } & \multirow[b]{2}{*}{$\begin{array}{l}\text { Sampled at } \\
(\mathrm{cm})\end{array}$} & \multicolumn{3}{|c|}{ NRM } & \multicolumn{3}{|c|}{50 oersted } \\
\hline & & & & Decl. & Incl. & $\begin{array}{l}\text { Intensity } \\
\text { emu/gm }\end{array}$ & Decl. & Incl. & $\begin{array}{l}\text { Intensity } \\
\text { emu/gm }\end{array}$ \\
\hline 18 & 4 & 4 & 50 & 156.5 & -45.1 & $0.3213 \times 10^{-5}$ & 98.6 & -87.3 & $0.1298 \times 10^{-4}$ \\
\hline 18 & 4 & 4 & 100 & 77.5 & -56.5 & $0.2303 \times 10^{-5}$ & 58.5 & -60.8 & $0.1523 \times 10^{-5}$ \\
\hline 18 & 5 & 2 & 7 & 203.9 & 17.7 & $0.9159 \times 10^{-6}$ & 224.4 & -35.5 & $0.6934 \times 10^{-6}$ \\
\hline 18 & 5 & 2 & 48 & 197.0 & 7.3 & $0.2480 \times 10^{-5}$ & 174.0 & 13.0 & $0.6605 \times 10^{-6}$ \\
\hline 18 & 5 & 2 & 127 & 195.4 & 29.5 & $0.2068 \times 10^{-5}$ & 188.8 & 35.3 & $0.5866 \times 10^{-6}$ \\
\hline 18 & 6 & 1 & 15 & 106.6 & 38.0 & $0.2945 \times 10^{-5}$ & 86.3 & 44.9 & $0.2667 \times 10^{-5}$ \\
\hline 18 & 6 & 1 & 100 & 193.1 & -2.2 & $0.2211 \times 10^{-5}$ & 205.6 & 18.5 & $0.1014 \times 10^{-5}$ \\
\hline 18 & 6 & 6 & 5 & 140.0 & 34.4 & $0.1946 \times 10^{-5}$ & 84.4 & 64.6 & $0.9542 \times 10^{-6}$ \\
\hline 18 & 6 & 6 & 50 & 177.0 & 33.7 & $0.3812 \times 10^{-5}$ & 216.9 & 61.9 & $0.1903 \times 10^{-5}$ \\
\hline *18 & 6 & 6 & $75-7$ & 162.0 & 43.7 & $0.1382 \times 10^{-5}$ & 146.4 & 77.0 & $0.1198 \times 10^{-5}$ \\
\hline 18 & 6 & 6 & 100 & 233.1 & 36.6 & $0.2427 \times 10^{-5}$ & 249.5 & 47.3 & $0.1665 \times 10^{-5}$ \\
\hline$* 19$ & 1 & 2 & $2-4$ & 196.3 & 2.9 & $0.7059 \times 10^{-5}$ & 250.6 & 48.8 & $0.2677 \times 10^{-5}$ \\
\hline 19 & 3 & 1 & 50 & 225.7 & -65.4 & $0.7663 \times 10^{-5}$ & 223.2 & -76.9 & $0.6776 \times 10^{-5}$ \\
\hline 19 & 3 & 1 & 105 & 155.7 & -50.9 & $0.9160 \times 10^{-5}$ & 99.8 & -56.1 & $0.4461 \times 10^{-5}$ \\
\hline 19 & 3 & 2 & 14 & 206.2 & 39.1 & $0.5107 \times 10^{-5}$ & 223.2 & 35.4 & $0.2561 \times 10^{-5}$ \\
\hline 19 & 3 & 2 & 50 & 199.6 & -15.8 & $0.1860 \times 10^{-4}$ & 207.2 & -37.8 & $0.9891 \times 10^{-5}$ \\
\hline 19 & 3 & 2 & 100 & 233.6 & -45.6 & $0.1666 \times 10^{-4}$ & 100.7 & -66.4 & $0.8179 \times 10^{-5}$ \\
\hline 19 & 3 & 3 & 13 & 184.5 & 43.3 & $0.8833 \times 10^{-5}$ & 110.1 & 44.7 & $0.1375 \times 10^{-5}$ \\
\hline 19 & 3 & 3 & 100 & 208.1 & 24.8 & $0.8000 \times 10^{-5}$ & 302.8 & 34.6 & $0.1827 \times 10^{-5}$ \\
\hline 19 & 3 & 4 & 13 & 172.2 & 8.2 & $0.1421 \times 10^{-4}$ & 169.8 & -6.2 & $0.4907 \times 10^{-5}$ \\
\hline 19 & 3 & 4 & 100 & 107.4 & -58.5 & $0.8841 \times 10^{-5}$ & 41.0 & -57.0 & $0.6281 \times 10^{-5}$ \\
\hline 19 & 3 & 5 & 10 & 121.1 & -19.7 & $0.7936 \times 10^{-5}$ & 87.2 & -46.0 & $0.5576 \times 10^{-5}$ \\
\hline 19 & 3 & 5 & 130 & 208.0 & 9.2 & $0.6194 \times 10^{-5}$ & 344.9 & 46.9 & $0.2788 \times 10^{-5}$ \\
\hline 19 & 3 & 6 & 12 & 148.2 & 19.4 & $0.9194 \times 10^{-5}$ & 85.4 & 56.0 & $0.3398 \times 10^{-5}$ \\
\hline 19 & 3 & 6 & 105 & 183.3 & 19.3 & $0.6052 \times 10^{-5}$ & 236.4 & 28.4 & $0.1045 \quad 10^{-5}$ \\
\hline 19 & 3 & 6 & 130 & 230.6 & 35.2 & $0.7140 \times 10^{-5}$ & 294.9 & 12.7 & $0.2638 \times 10^{-5}$ \\
\hline 19 & 4 & 2 & 1 & 311.2 & -28.7 & $0.2007 \times 10^{-4}$ & 319.1 & -21.3 & $0.1819 \times 10^{-4}$ \\
\hline 19 & 4 & 2 & 23 & 219.2 & 51.7 & $0.5802 \times 10^{-5}$ & 218.4 & 63.5 & $0.3877 \times 10^{-5}$ \\
\hline 19 & 4 & 2 & 100 & 129.7 & -10.6 & $0.1592 \times 10^{-4}$ & 125.5 & -15.6 & $0.1190 \times 10^{-4}$ \\
\hline
\end{tabular}


TABLE 1-Continued

\begin{tabular}{|c|c|c|c|c|c|c|c|c|c|}
\hline \multirow[b]{2}{*}{ Hole } & \multirow[b]{2}{*}{ Core } & \multirow[b]{2}{*}{ Section } & \multirow[b]{2}{*}{$\begin{array}{l}\text { Sampled at } \\
\text { (cm) }\end{array}$} & \multirow[b]{2}{*}{ Decl. } & \multicolumn{2}{|c|}{ NRM } & \multicolumn{3}{|c|}{50 oersted } \\
\hline & & & & & Incl. & $\begin{array}{l}\text { Intensity } \\
\text { emu/gm }\end{array}$ & Decl. & Incl. & $\begin{array}{l}\text { Intensity } \\
\text { emu/gm }\end{array}$ \\
\hline 19 & 5 & 2 & 1 & 189.0 & -47.2 & $0.4409 \times 10^{-5}$ & 161.5 & -80.1 & $0.8539 \times 10^{-6}$ \\
\hline 19 & 5 & 2 & 13 & 175.9 & 3.5 & $0.4569 \times 10^{-5}$ & 168.0 & -12.4 & $0.1607 \times 10^{-5}$ \\
\hline 19 & 5 & 2 & 50 & 173.1 & 34.7 & $0.1183 \times 10^{-4}$ & 155.7 & 37.2 & $0.8153 \times 10^{-5}$ \\
\hline 19 & 5 & 2 & 100 & 202.8 & 52.1 & $0.4586 \times 10^{-5}$ & 213.6 & 55.9 & $0.3957 \times 10^{-5}$ \\
\hline 19 & 5 & 3 & 3 & 169.3 & 34.9 & $0.1812 \times 10^{-4}$ & 151.1 & 37.8 & $0.1106 \times 10^{-5}$ \\
\hline 19 & 5 & 3 & 8 & 127.8 & 71.2 & $0.6653 \times 10^{-5}$ & 67.6 & 53.2 & $0.6310 \times 10^{-5}$ \\
\hline 19 & 5 & 3 & 51 & 153.7 & 20.0 & $0.1484 \times 10^{-4}$ & 138.3 & 33.2 & $0.9011 \times 10^{-5}$ \\
\hline 19 & 5 & 3 & 100 & 183.9 & 61.0 & $0.5072 \times 10^{-5}$ & 82.7 & 66.0 & $0.3570 \times 10^{-5}$ \\
\hline 19 & 5 & 4 & 19 & 195.2 & -78.5 & $0.5469 \times 10^{-5}$ & 80.9 & -74.5 & $0.3639 \times 10^{-5}$ \\
\hline 19 & 5 & 4 & 50 & 194.9 & -6.7 & $0.1022 \times 10^{-4}$ & 192.7 & -12.2 & $0.4276 \times 10^{-5}$ \\
\hline 19 & 5 & 4 & 100 & 133.9 & -33.4 & $0.8778 \times 10^{-5}$ & 119.6 & -49.2 & $0.5503 \times 10^{-5}$ \\
\hline 19 & 5 & 5 & 12 & 31.3 & -73.6 & $0.6553 \times 10^{-5}$ & 24.1 & -65.2 & $0.5193 \times 10^{-5}$ \\
\hline 19 & 5 & 5 & 50 & 218.9 & -20.1 & $0.9402 \times 10^{-5}$ & 241.1 & -48.8 & $0.5962 \times 10^{-5}$ \\
\hline 19 & 5 & 5 & 100 & 140.8 & 30.9 & $0.6848 \times 10^{-5}$ & 226.7 & 44.3 & $0.4959 \times 10^{-5}$ \\
\hline 19 & 5 & 6 & 3 & 194.6 & -43.0 & $0.1030 \times 10^{-4}$ & 115.3 & -72.2 & $0.3381 \times 10^{-5}$ \\
\hline 19 & 5 & 6 & 10 & 278.9 & -45.9 & $0.6605 \times 10^{-5}$ & 310.1 & -43.6 & $0.3487 \times 10^{-5}$ \\
\hline 19 & 5 & 6 & 50 & 207.4 & 45.9 & $0.8984 \times 10^{-5}$ & 286.1 & 62.3 & $0.4301 \times 10^{-5}$ \\
\hline 19 & 5 & 6 & 100 & 163.9 & 60.4 & $0.5249 \times 10^{-5}$ & 146.7 & 76.4 & $0.3023 \times 10^{-5}$ \\
\hline 19 & 7 & 1 & 10 & 214.8 & 30.2 & $0.9405 \times 10^{-5}$ & 175.8 & 71.3 & $0.6385 \times 10^{-5}$ \\
\hline 19 & 7 & 1 & 50 & 175.1 & -1.4 & $0.9867 \times 10^{-5}$ & 146.6 & 40.2 & $0.2912 \times 10^{-5}$ \\
\hline 19 & 7 & 1 & 100 & 203.4 & 27.7 & $0.5969 \times 10^{-5}$ & 228.7 & 50.7 & $0.3701 \times 10^{-5}$ \\
\hline 19 & 7 & 2 & 11 & 213.6 & -38.0 & $0.8690 \times 10^{-5}$ & 220.1 & -31.0 & $0.3788 \times 10^{-5}$ \\
\hline 19 & 7 & 2 & 50 & 157.8 & -14.4 & $0.1328 \times 10^{-4}$ & 144.0 & -25.6 & $0.8802 \times 10^{-5}$ \\
\hline 19 & 7 & 2 & 100 & 120.2 & -57.8 & $0.5563 \times 10^{-5}$ & 93.6 & -61.0 & $0.3912 \times 10^{-5}$ \\
\hline 19 & 7 & 3 & 2 & 265.1 & -35.2 & $0.9947 \times 10^{-5}$ & 296.8 & -41.7 & $0.7003 \times 10^{-5}$ \\
\hline 19 & 7 & 3 & 11 & 156.8 & -40.5 & $0.8824 \times 10^{-5}$ & 143.6 & -51.3 & $0.4962 \times 10^{-5}$ \\
\hline 19 & 7 & 3 & 100 & 80.5 & -36.4 & $0.1111 \times 10^{-4}$ & 67.0 & -35.7 & $0.8601 \times 10^{-5}$ \\
\hline 19 & 7 & 4 & 4 & 171.6 & 35.5 & $0.1524 \times 10^{-4}$ & 255.5 & -18.3 & $0.3098 \times 10^{-5}$ \\
\hline 19 & 7 & 4 & 50 & 193.8 & 35.9 & $0.1104 \times 10^{-4}$ & 340.0 & 60.8 & $0.4950 \times 10^{-5}$ \\
\hline
\end{tabular}


TABLE 1-Continued

\begin{tabular}{|c|c|c|c|c|c|c|c|c|c|}
\hline \multirow[b]{2}{*}{ Hole } & \multirow[b]{2}{*}{ Core } & \multirow[b]{2}{*}{ Section } & \multirow[b]{2}{*}{$\begin{array}{l}\text { Sampled at } \\
\text { (cm) }\end{array}$} & \multicolumn{3}{|c|}{ NRM } & \multicolumn{3}{|c|}{50 oersted } \\
\hline & & & & Decl. & Incl. & $\begin{array}{l}\text { Intensity } \\
\text { emu/gm }\end{array}$ & Decl. & Incl. & $\begin{array}{l}\text { Intensity } \\
\text { emu/gm }\end{array}$ \\
\hline 19 & 7 & 4 & 100 & 234.5 & 21.7 & $0.6918 \times 10^{-5}$ & 264.4 & 40.8 & $0.4588 \times 10^{-5}$ \\
\hline 19 & 7 & 5 & 3 & 219.4 & -18.1 & $0.1040 \times 10^{-4}$ & 348.5 & -74.2 & $0.2501 \times 10^{-5}$ \\
\hline 19 & 7 & 5 & 9 & 220.7 & -48.6 & $0.1142 \times 10^{-4}$ & 244.6 & -56.4 & $0.5188 \times 10^{-5}$ \\
\hline 19 & 7 & 5 & 100 & 197.1 & 36.8 & $0.2898 \times 10^{-5}$ & 22.3 & 33.8 & $0.1765 \times 10^{-5}$ \\
\hline 19 & 7 & 6 & 3 & 199.8 & -43.0 & $0.1225 \times 10^{-4}$ & 121.3 & 36.2 & $0.1928 \times 10^{-5}$ \\
\hline 19 & 7 & 6 & 11 & 171.0 & 15.3 & $0.4810 \times 10^{-5}$ & 152.0 & 62.2 & $0.3323 \times 10^{-5}$ \\
\hline 19 & 7 & 6 & 50 & 151.9 & 8.3 & $0.3286 \times 10^{-5}$ & 119.5 & -18.3 & $0.3119 \times 10^{-6}$ \\
\hline 19 & 7 & 6 & 62 & 238.7 & -24.3 & $0.1063 \times 10^{-5}$ & 284.8 & 60.2 & $0.3773 \times 10^{-6}$ \\
\hline 19 & 8 & 2 & 11 & 64.1 & 44.9 & $0.4631 \times 10^{-5}$ & 62.2 & 30.7 & $0.2102 \times 10^{-5}$ \\
\hline 19 & 8 & 2 & 46 & 221.7 & -10.9 & $0.7781 \times 10^{-5}$ & 225.0 & -26.5 & $0.4849 \times 10^{-5}$ \\
\hline 19 & 8 & 2 & 102 & 273.0 & -46.8 & $0.4872 \times 10^{-5}$ & 287.2 & -33.3 & $0.5513 \times 10^{-5}$ \\
\hline 19 & 9 & 6 & 12 & 146.5 & -37.5 & $0.5664 \times 10^{-5}$ & 156.2 & -72.2 & $0.9282 \times 10^{-5}$ \\
\hline 19 & 9 & 6 & 50 & 209.1 & -19.0 & $0.1280 \times 10^{-4}$ & 219.1 & -33.8 & $0.4417 \times 10^{-5}$ \\
\hline 19 & 9 & 6 & 70 & 317.0 & -33.9 & $0.7935 \times 10^{-5}$ & 338.1 & -35.6 & $0.3454 \times 10^{-5}$ \\
\hline $20 \mathrm{~A}$ & 2 & 1 & 54 & 210.9 & -64.5 & $0.4891 \times 10^{-5}$ & 270.4 & -25.7 & $0.7967 \times 10^{-5}$ \\
\hline $20 \mathrm{~A}$ & 2 & 1 & 100 & 167.9 & -11.4 & $0.5863 \times 10^{-5}$ & 169.9 & 2.0 & $0.2897 \times 10^{-5}$ \\
\hline $20 \mathrm{~A}$ & 2 & 2 & 13 & 155.9 & -78.7 & $0.1488 \times 10^{-5}$ & 112.4 & -11.5 & $0.2910 \times 10^{-5}$ \\
\hline$* 20 \mathrm{~A}$ & 2 & 2 & $50-2$ & 216.3 & -14.0 & $0.1419 \times 10^{-5}$ & 295.9 & 22.7 & $0.4284 \times 10^{-6}$ \\
\hline $20 \mathrm{~A}$ & 2 & 2 & 120 & 219.9 & 2.2 & $0.3711 \times 10^{-5}$ & 201.8 & 72.3 & $0.1332 \times 10^{-5}$ \\
\hline $20 \mathrm{~A}$ & 2 & 3 & 14 & 168.0 & 17.5 & $0.2752 \times 10^{-5}$ & 43.9 & 71.4 & $0.2304 \times 10^{-5}$ \\
\hline $20 \mathrm{~B}$ & 1 & 1 & 10 & 113.7 & -47.2 & $0.7503 \times 10^{-5}$ & 293.3 & -29.1 & $0.5105 \times 10^{-5}$ \\
\hline $20 \mathrm{~B}$ & 1 & 1 & 50 & 165.6 & -10.3 & $0.2130 \times 10^{-4}$ & 147.9 & 8.3 & $0.3827 \times 10^{-5}$ \\
\hline$* 20 \mathrm{~B}$ & 1 & 2 & $7-9$ & 207.1 & -16.7 & $0.7617 \times 10^{-5}$ & 281.9 & -40.6 & $0.4168 \times 10^{-5}$ \\
\hline $20 \mathrm{~B}$ & 1 & 2 & 14 & 179.5 & 6.8 & $0.4395 \times 10^{-5}$ & 303.5 & -39.8 & $0.1424 \times 10^{-5}$ \\
\hline $20 \mathrm{~B}$ & 1 & 2 & 50 & 200.4 & 9.1 & $0.2240 \times 10^{-4}$ & 211.2 & -28.8 & $0.5591 \times 10^{-5}$ \\
\hline $20 \mathrm{~B}$ & 1 & 2 & 102 & 150.0 & -0.4 & $0.9467 \times 10^{-5}$ & 174.2 & 11.6 & $0.1425 \times 10^{-5}$ \\
\hline $20 \mathrm{~B}$ & 1 & 3 & 4 & 165.7 & 12.9 & $0.2239 \times 10^{-4}$ & 129.4 & -32.4 & $0.2128 \times 10^{-5}$ \\
\hline $20 \mathrm{~B}$ & 1 & 3 & 12 & 184.4 & -39.2 & $0.5292 \times 10^{-5}$ & 113.2 & -48.4 & $0.1001 \times 10^{-5}$ \\
\hline $20 \mathrm{~B}$ & 1 & 3 & 50 & 189.3 & 3.0 & $0.1793 \times 10^{-4}$ & 185.3 & -34.7 & $0.3701 \times 10^{-5}$ \\
\hline
\end{tabular}


TABLE 1-Continued

\begin{tabular}{|c|c|c|c|c|c|c|c|c|c|}
\hline \multirow[b]{2}{*}{ Hole } & \multirow[b]{2}{*}{ Core } & \multirow[b]{2}{*}{ Section } & \multirow[b]{2}{*}{$\begin{array}{l}\text { Sampled at } \\
(\mathrm{cm})\end{array}$} & \multicolumn{3}{|c|}{ NRM } & \multicolumn{3}{|c|}{50 oersted } \\
\hline & & & & Decl. & Incl. & $\begin{array}{l}\text { Intensity } \\
\text { emu/gm }\end{array}$ & Decl. & Incl. & $\begin{array}{l}\text { Intensity } \\
\text { emu/gm }\end{array}$ \\
\hline $20 \mathrm{~B}$ & 1 & 3 & 100 & 163.3 & -30.6 & $0.8165 \times 10^{-5}$ & 149.1 & -45.2 & $0.2864 \times 10^{-5}$ \\
\hline $20 \mathrm{~B}$ & 1 & 4 & 3 & 207.1 & -2.8 & $0.2106 \times 10^{-4}$ & 112.3 & -75.2 & $0.4515 \times 10^{-5}$ \\
\hline $20 \mathrm{~B}$ & 1 & 4 & 13 & 150.6 & -47.1 & $0.9214 \times 10^{-5}$ & 112.1 & -69.2 & $0.3432 \times 10^{-5}$ \\
\hline $20 \mathrm{~B}$ & 1 & 4 & 100 & 216.6 & -14.7 & $0.9180 \times 10^{-5}$ & 111.9 & -81.2 & $0.3831 \times 10^{-5}$ \\
\hline $20 \mathrm{~B}$ & 1 & 5 & $0-2$ & 183.0 & -36.6 & $0.2722 \times 10^{-4}$ & 132.1 & -71.0 & $0.3987 \times 10^{-5}$ \\
\hline $20 \mathrm{~B}$ & 1 & 5 & 50 & 185.3 & -20.4 & $0.1927 \times 10^{-4}$ & 324.9 & -55.8 & $0.6127 \times 10^{-5}$ \\
\hline $20 \mathrm{~B}$ & 1 & 5 & 110 & 177.5 & -17.6 & $0.6843 \times 10^{-5}$ & 50.9 & -50.4 & $0.5974 \times 10^{-6}$ \\
\hline $20 \mathrm{~B}$ & 1 & 6 & 1 & 205.7 & -2.3 & $0.2732 \times 10^{-4}$ & 182.6 & -39.0 & $0.3327 \times 10^{-5}$ \\
\hline $20 \mathrm{~B}$ & 1 & 6 & 100 & 256.9 & -35.2 & $0.1017 \times 10^{-4}$ & 266.4 & -2.5 & $0.6197 \times 10^{-5}$ \\
\hline $20 \mathrm{C}$ & 1 & 1 & 50 & 178.7 & -24.0 & $0.2347 \times 10^{-4}$ & 185.8 & -39.6 & $0.1039 \times 10^{-4}$ \\
\hline $20 \mathrm{C}$ & 1 & 1 & 60 & 179.4 & 27.2 & $0.6251 \times 10^{-5}$ & 170.4 & 53.4 & $0.5701 \times 10^{-5}$ \\
\hline $20 \mathrm{C}$ & 1 & 2 & 146 & 306.8 & 41.2 & $0.3867 \times 10^{-5}$ & 353.2 & 40.8 & $0.6109 \times 10^{-5}$ \\
\hline $20 \mathrm{C}$ & 1 & 3 & 68 & 247.6 & 14.4 & $0.1542 \times 10^{-4}$ & 284.4 & 23.4 & $0.1070 \times 10^{-4}$ \\
\hline $20 \mathrm{C}$ & 1 & 3 & 76 & 234.0 & 26.8 & $0.1720 \times 10^{-4}$ & 116.8 & 31.4 & $0.1478 \times 10^{-4}$ \\
\hline $20 \mathrm{C}$ & 1 & 5 & 3 & 105.6 & 12.3 & $0.7800 \times 10^{-5}$ & 68.5 & 32.9 & $0.7332 \times 10^{-5}$ \\
\hline $20 \mathrm{C}$ & 1 & 5 & 105 & 200.9 & -41.4 & $0.1179 \times 10^{-4}$ & 198.6 & -66.0 & $0.6036 \times 10^{-5}$ \\
\hline $20 \mathrm{C}$ & 1 & 6 & 22 & 174.5 & 0.9 & $0.2537 \times 10^{-4}$ & 178.3 & 3.2 & $0.1067 \times 10^{-4}$ \\
\hline $20 \mathrm{C}$ & 1 & 6 & 50 & 142.1 & -46.5 & $0.1387 \times 10^{-4}$ & 85.1 & -48.9 & $0.8655 \times 10^{-5}$ \\
\hline $20 \mathrm{C}$ & 1 & 6 & 99 & 181.3 & -5.5 & $0.7148 \times 10^{-5}$ & 147.4 & -50.6 & $0.1273 \times 10^{-5}$ \\
\hline $20 \mathrm{C}$ & 2 & 3 & 68 & 204.9 & -4.9 & $0.4258 \times 10^{-5}$ & 198.9 & 8.9 & $0.2716 \times 10^{-5}$ \\
\hline $20 \mathrm{C}$ & 2 & 4 & 12 & 223.1 & -4.3 & $0.2159 \times 10^{-5}$ & 264.2 & 6.2 & $0.1464 \times 10^{-5}$ \\
\hline $20 \mathrm{C}$ & 2 & 4 & 50 & 148.6 & -5.2 & $0.2132 \times 10^{-5}$ & 327.1 & -25.2 & $0.5972 \times 10^{-6}$ \\
\hline $20 \mathrm{C}$ & 2 & 4 & 112 & 222.1 & 20.0 & $0.1459 \times 10^{-5}$ & 269.5 & 15.5 & $0.6307 \times 10^{-6}$ \\
\hline $20 \mathrm{C}$ & 3 & 1 & 13 & 166.6 & 30.5 & $0.8725 \times 10^{-5}$ & 161.4 & 25.8 & $0.8435 \times 10^{-5}$ \\
\hline $20 \mathrm{C}$ & 3 & 1 & 50 & 169.2 & -29.2 & $0.4055 \times 10^{-4}$ & 202.4 & -26.2 & $0.5790 \times 10^{-5}$ \\
\hline $20 \mathrm{C}$ & 3 & 3 & 19 & 188.2 & 20.9 & $0.9178 \times 10^{-5}$ & 87.0 & 59.1 & $0.3621 \times 10^{-5}$ \\
\hline $20 \mathrm{C}$ & 3 & 4 & 22 & 211.1 & -43.4 & $0.6558 \times 10^{-5}$ & 3.2 & -50.1 & $0.1771 \times 10^{-5}$ \\
\hline $20 \mathrm{C}$ & 3 & 4 & 38 & 193.2 & -19.8 & $0.2625 \times 10^{-4}$ & 245.8 & -35.4 & $0.7304 \times 10^{-5}$ \\
\hline $20 \mathrm{C}$ & 3 & 4 & 77 & 197.0 & 15.3 & $0.9409 \times 10^{-5}$ & 199.8 & 33.1 & $0.2800 \times 10^{-5}$ \\
\hline
\end{tabular}


TABLE 1-Continued

\begin{tabular}{|c|c|c|c|c|c|c|c|c|c|}
\hline \multirow[b]{2}{*}{ Hole } & \multirow[b]{2}{*}{ Core } & \multirow[b]{2}{*}{ Section } & \multirow[b]{2}{*}{$\begin{array}{l}\text { Sampled at } \\
(\mathrm{cm})\end{array}$} & \multicolumn{3}{|c|}{ NRM } & \multicolumn{3}{|c|}{50 oersted } \\
\hline & & & & Decl. & Incl. & $\begin{array}{l}\text { Intensity } \\
\text { emu/gm }\end{array}$ & Decl. & Incl. & $\begin{array}{l}\text { Intensity } \\
\text { emu/gm }\end{array}$ \\
\hline $20 \mathrm{C}$ & 3 & 5 & 62 & 211.3 & 32.0 & $0.6928 \times 10^{-5}$ & 218.3 & 18.0 & $0.6473 \times 10^{-5}$ \\
\hline $20 \mathrm{C}$ & 3 & 5 & 122 & 176.2 & -75.7 & $0.9665 \times 10^{-5}$ & 351.3 & -80.6 & $0.5684 \times 10^{-5}$ \\
\hline $20 \mathrm{C}$ & 3 & 6 & 17 & 202.8 & 65.2 & $0.1108 \times 10^{-4}$ & 325.3 & 18.5 & $0.3406 \times 10^{-5}$ \\
\hline $20 \mathrm{C}$ & 3 & 6 & 104 & 168.2 & 20.8 & $0.7547 \times 10^{-5}$ & 221.4 & 41.3 & $0.2823 \times 10^{-5}$ \\
\hline $20 \mathrm{C}$ & 4 & 2 & 21 & 167.0 & 69.9 & $0.1637 \times 10^{-4}$ & 132.8 & 56.0 & $0.7224 \times 10^{-5}$ \\
\hline $20 \mathrm{C}$ & 4 & 3 & 9 & 252.6 & 8.2 & $0.2629 \times 10^{-4}$ & 263.6 & 2.3 & $0.3236 \times 10^{-4}$ \\
\hline $20 \mathrm{C}$ & 4 & 3 & 100 & 287.8 & .18 .3 & $0.1174 \times 10^{-3}$ & 250.3 & -17.2 & $0.1131 \times 10^{-3}$ \\
\hline $20 \mathrm{C}$ & 5 & 2 & 13 & 277.0 & 3.2 & $0.1387 \times 10^{-3}$ & 259.5 & 4.6 & $0.1300 \times 10^{-3}$ \\
\hline $20 \mathrm{C}$ & 5 & 4 & 8 & 246.2 & 3.3 & $0.5265 \times 10^{-5}$ & 269.1 & -11.0 & $0.3200 \times 10^{-5}$ \\
\hline *20C & 5 & 4 & $60-62$ & 166.7 & 1.5 & $0.3578 \times 10^{-5}$ & 148.2 & 30.7 & $0.6745 \times 10^{-6}$ \\
\hline $20 \mathrm{C}$ & 5 & 4 & 100 & 355.3 & 2.2 & $0.3080 \times 10^{-5}$ & 216.4 & 30.9 & $0.5961 \times 10^{-6}$ \\
\hline $20 \mathrm{C}$ & 5 & 5 & 14 & 170.8 & 20.1 & $0.6394 \times 10^{-5}$ & 175.8 & -26.3 & $0.7696 \times 10^{-6}$ \\
\hline $20 \mathrm{C}$ & 5 & 5 & 50 & 178.6 & -13.1 & $0.7392 \times 10^{-5}$ & 185.6 & -15.9 & $0.5493 \times 10^{-6}$ \\
\hline $20 \mathrm{C}$ & 5 & 5 & 105 & 181.9 & 18.4 & $0.2392 \times 10^{-5}$ & 101.2 & 65.6 & $0.6194 \times 10^{-6}$ \\
\hline $20 \mathrm{C}$ & 5 & 6 & 11 & 170.1 & -6.1 & $0.2102 \times 10^{-5}$ & 148.7 & 25.4 & $0.5192 \times 10^{-6}$ \\
\hline $20 \mathrm{C}$ & 5 & 6 & 100 & 144.3 & -51.5 & $0.4119 \times 10^{-5}$ & 132.5 & -27.3 & $0.4498 \times 10^{-6}$ \\
\hline $20 \mathrm{C}$ & 6 & 4 & 2 & 192.8 & -4.2 & $0.8174 \times 10^{-5}$ & 63.1 & 73.1 & $0.1112 \times 10^{-5}$ \\
\hline $20 \mathrm{C}$ & 6 & 4 & 5 & 179.1 & 5.8 & $0.2250 \times 10^{-5}$ & 139.6 & 55.2 & $0.1295 \times 10^{-5}$ \\
\hline $20 \mathrm{C}$ & 6 & 4 & 85 & 190.5 & -10.8 & $\longrightarrow$ & 262.4 & 56.3 & \\
\hline $20 \mathrm{C}$ & 6 & 4 & 100 & 204.9 & -10.5 & $0.4681 \times 10^{-5}$ & 334.4 & 26.2 & $0.1095 \times 10^{-5}$ \\
\hline $20 \mathrm{C}$ & 6 & 4 & 112 & 62.1 & -17.6 & $0.5592 \times 10^{-5}$ & 39.8 & -17.4 & $0.5014 \times 10^{-5}$ \\
\hline $20 \mathrm{C}$ & 6 & 4 & 134 & 192.9 & 11.7 & $0.5597 \times 10^{-5}$ & 144.4 & 25.1 & $0.9621 \times 10^{-6}$ \\
\hline $20 \mathrm{C}$ & 6 & 5 & $0-2$ & 162.1 & -30.3 & $0.6371 \times 10^{-5}$ & 242.2 & -37.2 & $0.6302 \times 10^{-6}$ \\
\hline $20 \mathrm{C}$ & 6 & 5 & 8 & 182.3 & 6.9 & $0.6048 \times 10^{-5}$ & 333.6 & 40.9 & $0.4160 \times 10^{-6}$ \\
\hline $20 \mathrm{C}$ & 6 & 5 & 19 & 228.9 & 21.4 & $0.2693 \times 10^{-5}$ & 292.6 & 22.3 & $0.1135 \times 01^{-5}$ \\
\hline $20 \mathrm{C}$ & 6 & 5 & 30 & 288.2 & 53.9 & $0.5554 \times 10^{-5}$ & 319.4 & 44.3 & $0.4363 \times 10^{-5}$ \\
\hline $20 \mathrm{C}$ & 6 & 5 & 40 & 161.5 & 51.8 & $0.6914 \times 10^{-5}$ & 145.3 & 59.2 & $0.3261 \times 10^{-5}$ \\
\hline $20 \mathrm{C}$ & 6 & 5 & 75 & 343.2 & 77.3 & $0.1164 \times 10^{-4}$ & 3.2 & 71.3 & $0.9614 \times 10^{-5}$ \\
\hline $20 \mathrm{C}$ & 6 & 5 & 78 & 200.1 & 64.4 & $0.1719 \times 10^{-4}$ & 289.0 & 77.3 & $0.1261 \times 10^{-4}$ \\
\hline
\end{tabular}


TABLE 1-Continued

\begin{tabular}{|c|c|c|c|c|c|c|c|c|c|}
\hline \multirow[b]{2}{*}{ Hole } & \multirow[b]{2}{*}{ Core } & \multirow[b]{2}{*}{ Section } & \multirow[b]{2}{*}{$\begin{array}{l}\text { Sampled at } \\
\text { (cm) }\end{array}$} & \multicolumn{3}{|c|}{ NRM } & \multicolumn{3}{|c|}{50 oersted } \\
\hline & & & & Decl. & Incl. & $\begin{array}{l}\text { Intensity } \\
\mathrm{emu} / \mathrm{gm}\end{array}$ & Decl. & Incl. & $\begin{array}{l}\text { Intensity } \\
\text { emu/gm }\end{array}$ \\
\hline $20 \mathrm{C}$ & 6 & 5 & 100 & 138.0 & 72.4 & $0.1094 \times 10^{-4}$ & 130.1 & 86.0 & $0.7779 \times 10^{-5}$ \\
\hline $20 \mathrm{C}$ & 6 & 5 & 140 & 195.4 & -25.5 & $0.7973 \times 10^{-5}$ & 288.3 & -51.4 & $0.3462 \times 10^{-5}$ \\
\hline 21 & 2 & 6 & 143 & 194.4 & 34.4 & $0.6114 \times 10^{-5}$ & 327.2 & 40.3 & $0.1835 \times 10^{-5}$ \\
\hline 21 & 6 & 3 & 7 & 186.0 & -3.9 & $0.6767 \times 10^{-5}$ & 217.9 & -51.9 & $0.2017 \times 10^{-5}$ \\
\hline 21 & 6 & 4 & 2 & 142.9 & -34.7 & $0.7828 \times 10^{-5}$ & 101.8 & -50.2 & $0.2321 \times 10^{-5}$ \\
\hline 21 & 6 & 4 & 100 & 205.9 & -43.9 & $0.3435 \times 10^{-5}$ & 261.3 & -53.4 & $0.1881 \times 10^{-5}$ \\
\hline 21 & 6 & 5 & 3 & 163.5 & -19.7 & $0.9373 \times 10^{-5}$ & 152.7 & -46.6 & $0.5062 \times 10^{-5}$ \\
\hline 21 & 6 & 5 & 100 & 293.7 & -47.7 & $0.7274 \times 10^{-5}$ & 283.9 & -18.3 & $0.3287 \times 10^{-5}$ \\
\hline$* 21$ & 6 & 6 & $3-5$ & 156.7 & 41.9 & $0.9655 \times 10^{-5}$ & 147.8 & 45.5 & $0.6133 \times 10^{-5}$ \\
\hline 21 & 6 & 6 & 100 & 241.0 & -50.6 & $0.2291 \times 10^{-5}$ & 241.0 & -59.9 & $0.1756 \times 10^{-5}$ \\
\hline $21 \mathrm{~A}$ & 3 & 6 & 20 & 165.5 & 28.4 & $0.3620 \times 10^{-5}$ & 149.5 & 37.6 & $0.1851 \times 10^{-5}$ \\
\hline $21 \mathrm{~A}$ & 3 & 6 & 80 & 187.2 & 21.6 & $0.2759 \times 10^{-5}$ & 131.1 & 22.1 & $0.1308 \times 10^{-5}$ \\
\hline $21 \mathrm{~A}$ & 3 & 6 & 136 & 233.9 & 53.8 & $0.4372 \times 10^{-5}$ & 45.0 & 70.3 & $0.2370 \times 10^{-5}$ \\
\hline 22 & 1 & 6 & 60 & 218.9 & -14.1 & $0.2548 \times 10^{-5}$ & 235.3 & -26.3 & $0.1500 \times 10^{-5}$ \\
\hline 22 & 1 & 6 & 100 & 182.8 & -69.9 & $0.3759 \times 10^{-6}$ & 199.1 & -73.8 & $0.2808 \times 10^{-6}$ \\
\hline 22 & 4 & 2 & 55 & 254.3 & 16.4 & $0.2302 \times 10^{-6}$ & 242.2 & 19.6 & $0.2735 \times 10^{-6}$ \\
\hline 22 & 4 & 4 & 100 & 332.7 & -77.9 & $0.3570 \times 10^{-6}$ & 338.4 & -74.5 & $0.3757 \times 10^{-6}$ \\
\hline 22 & 4 & 5 & 100 & 133.8 & -35.5 & $0.1430 \times 10^{-5}$ & 130.3 & -35.7 & $0.1234 \times 10^{-5}$ \\
\hline 22 & 4 & 6 & 13 & 35.2 & -69.3 & $0.3452 \times 10^{-6}$ & 62.0 & -77.8 & $0.3583 \times 10^{-6}$ \\
\hline
\end{tabular}


Figures 1-13

Alternating field demagnetization curves for pilot specimens from Sites 13 through 21 . 


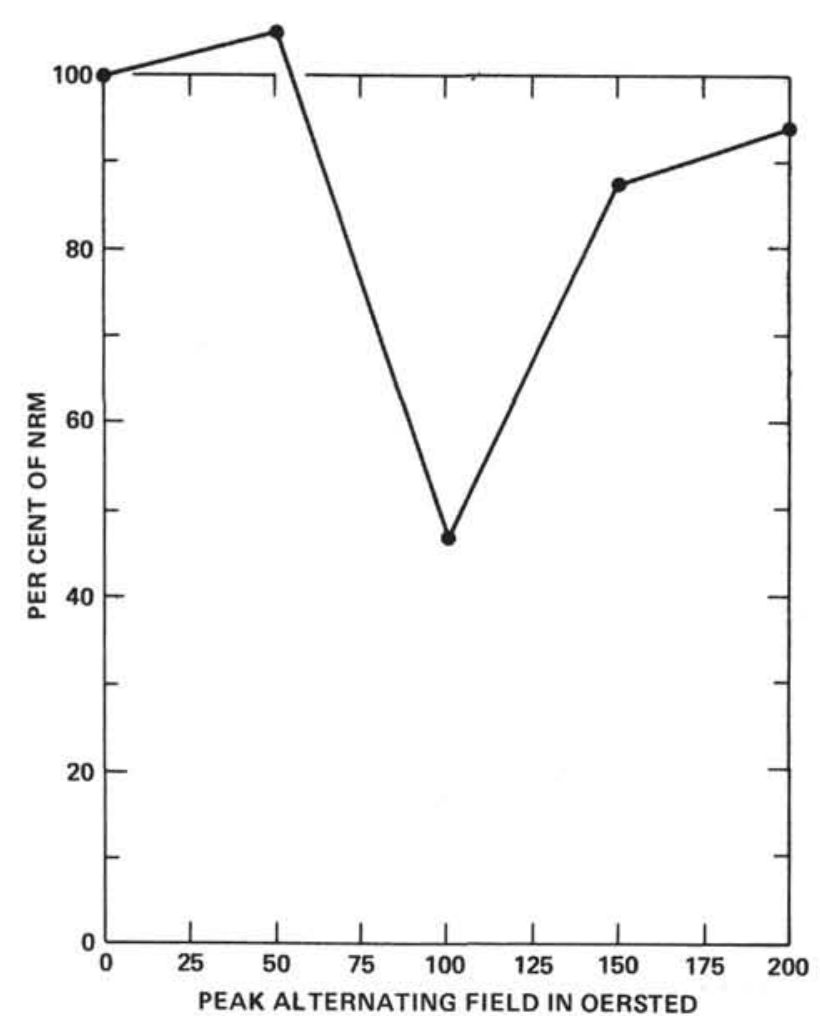

Figure 1. 3-13A-5-1, depth 49-51 cm.

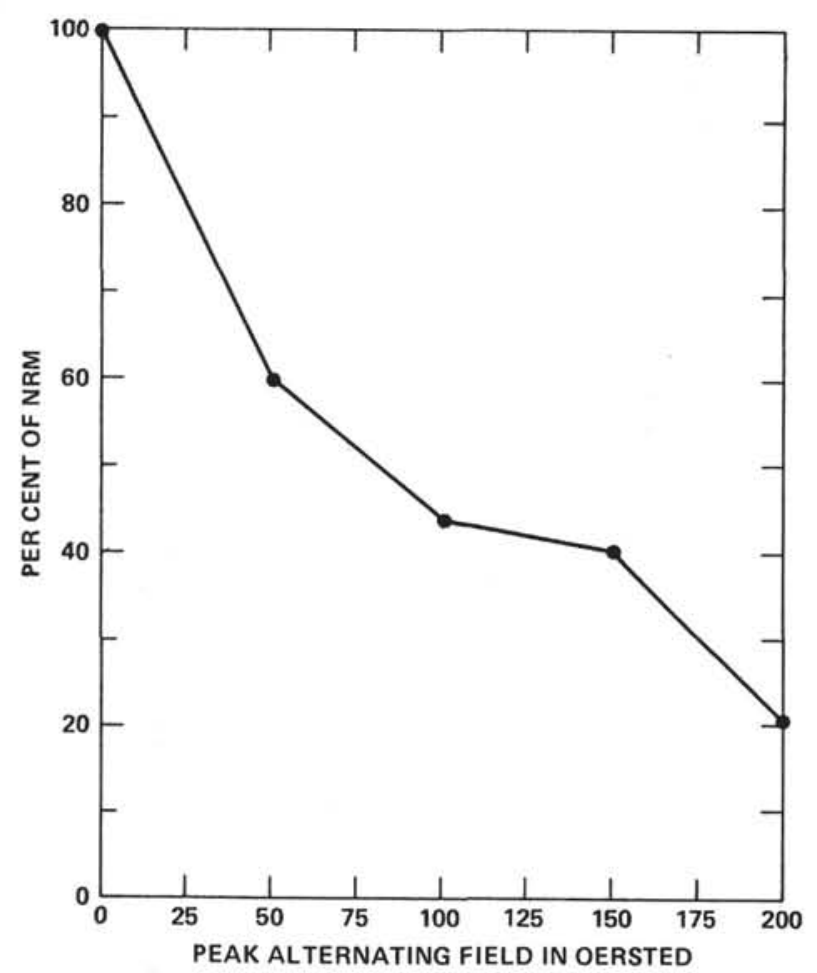

Figure 3. 3-15-4-3, depth $41-43 \mathrm{~cm}$.

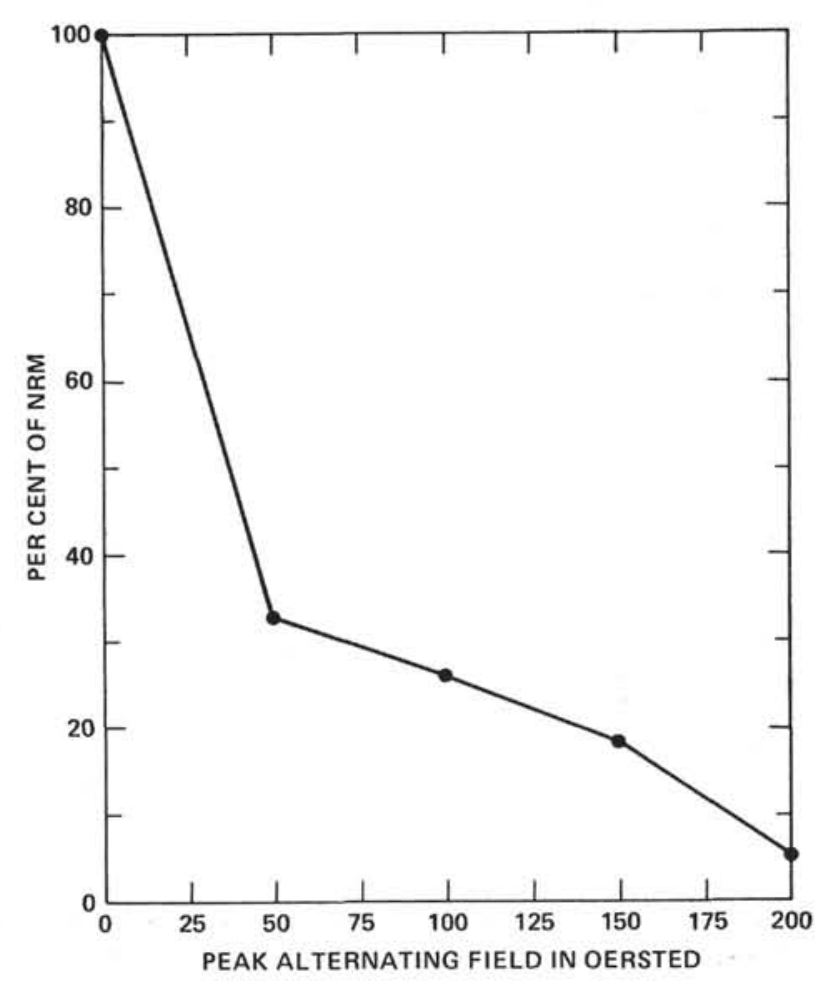

Figure 2. 3-14A-1-3, depth 50-52 cm.

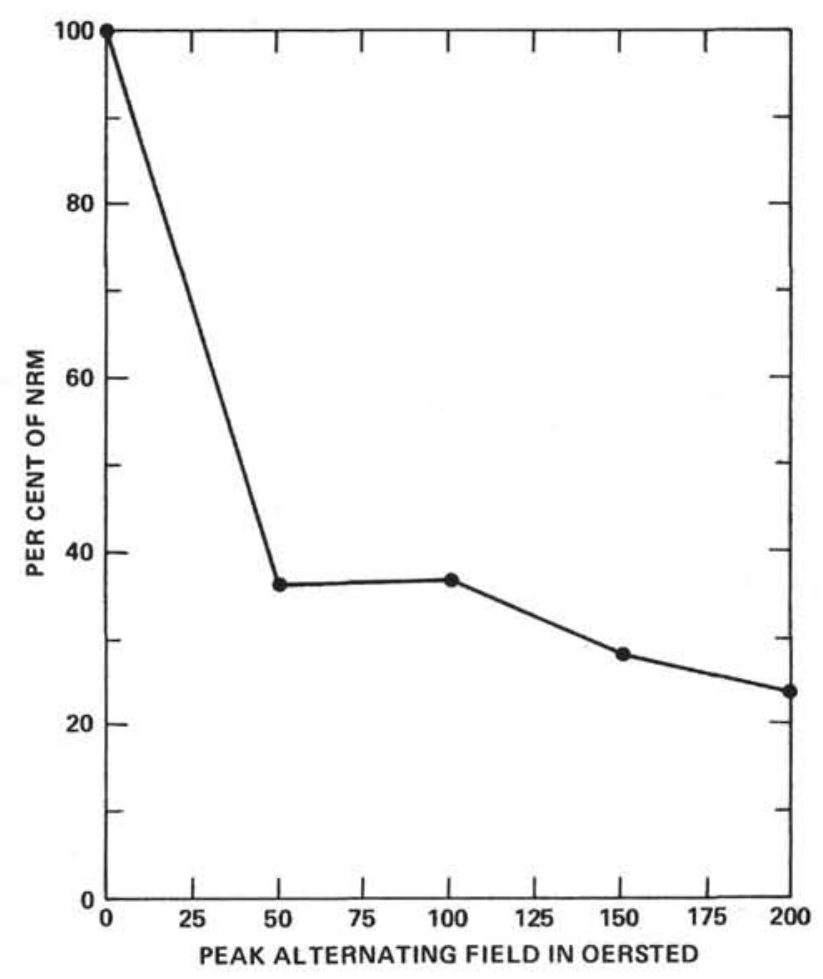

Figure 4. 3-16-2-6, depth 50-52 cm. 


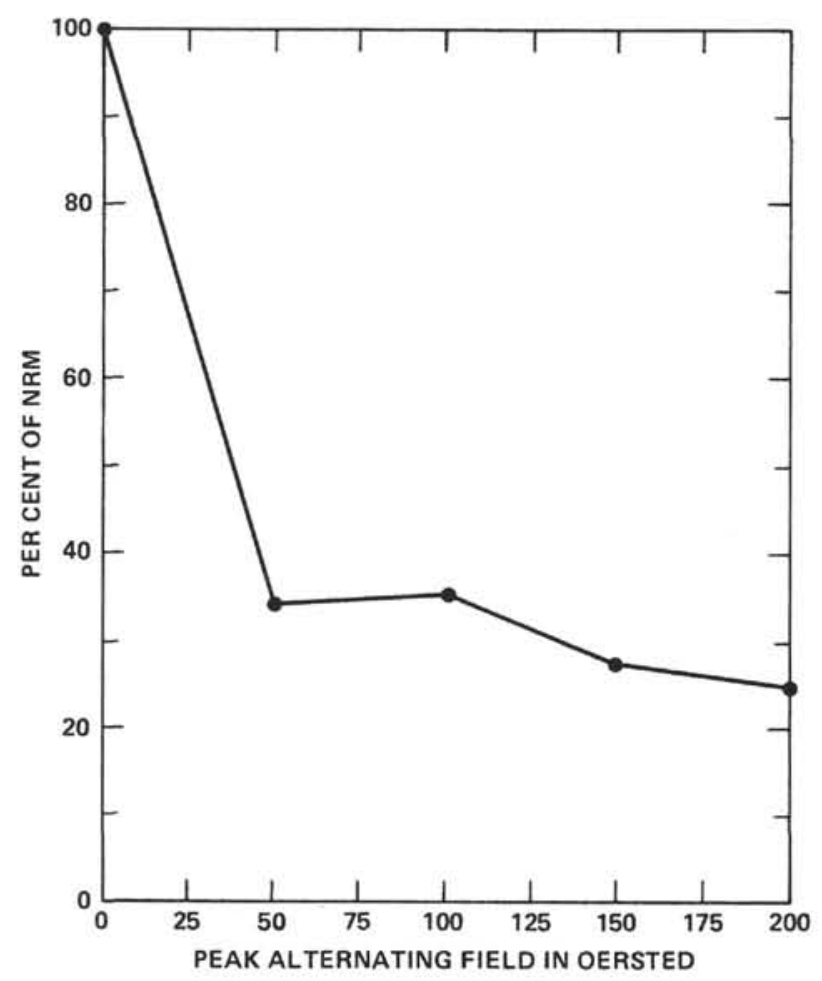

Figure 5. 3-17-2-3, depth $54-56 \mathrm{~cm}$.

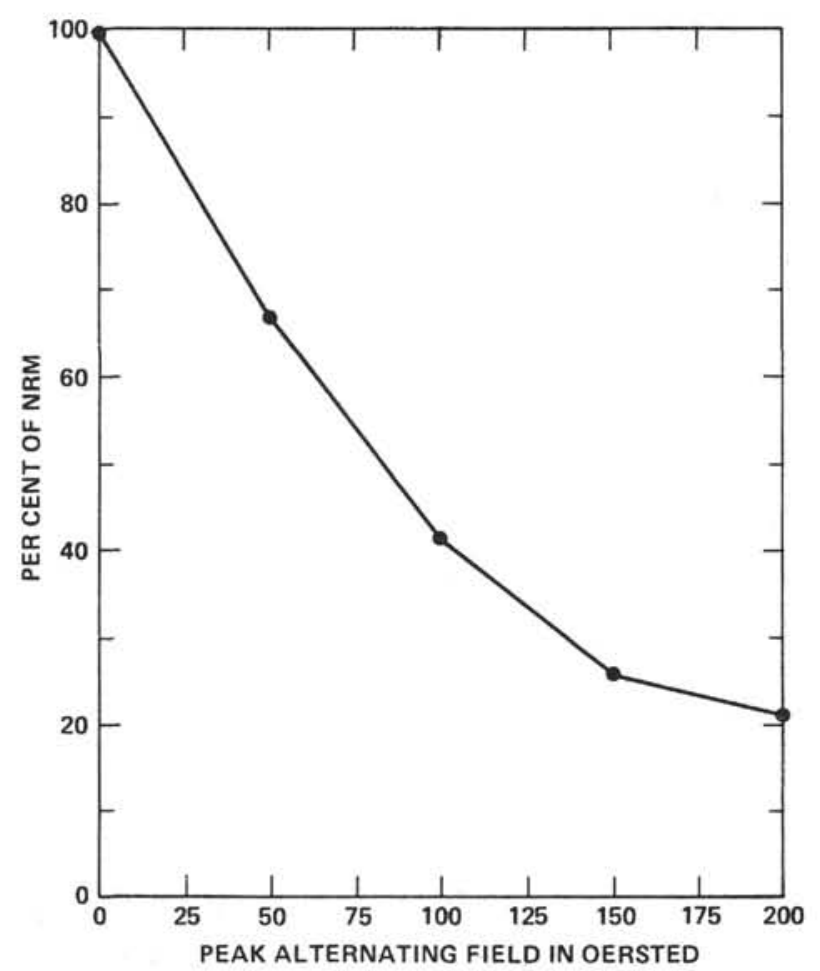

Figure 7. 3-17B-4-6, depth $53-55 \mathrm{~cm}$.

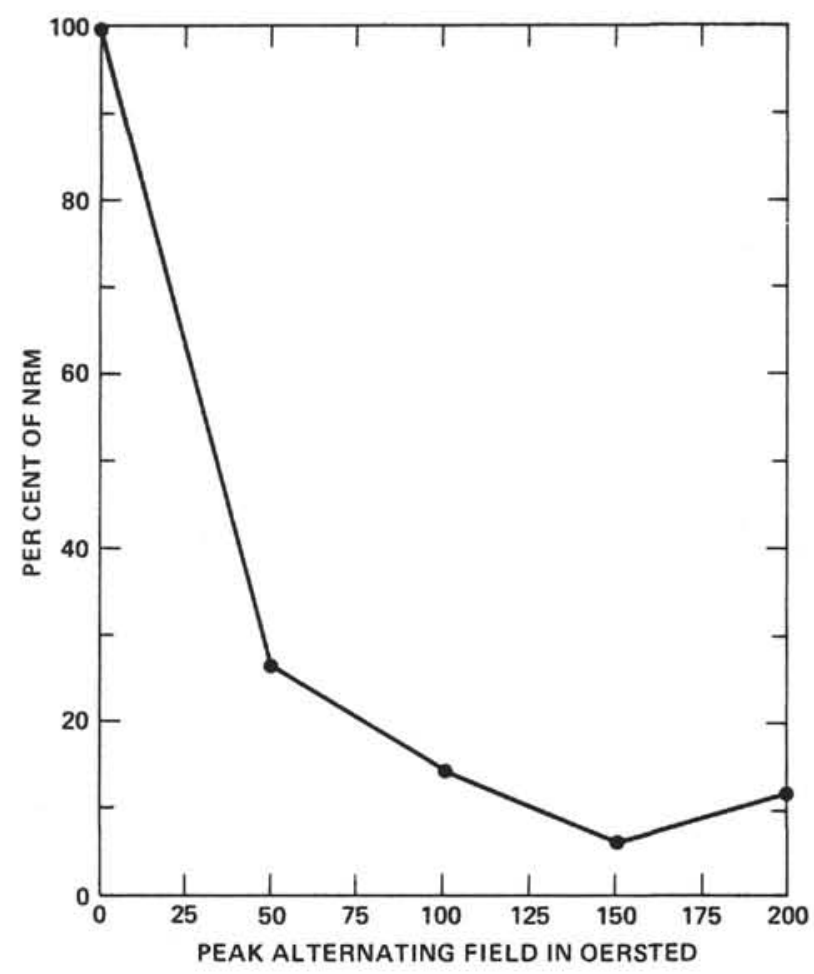

Figure 6. 3-17A-2-4, depth 50-52 cm.

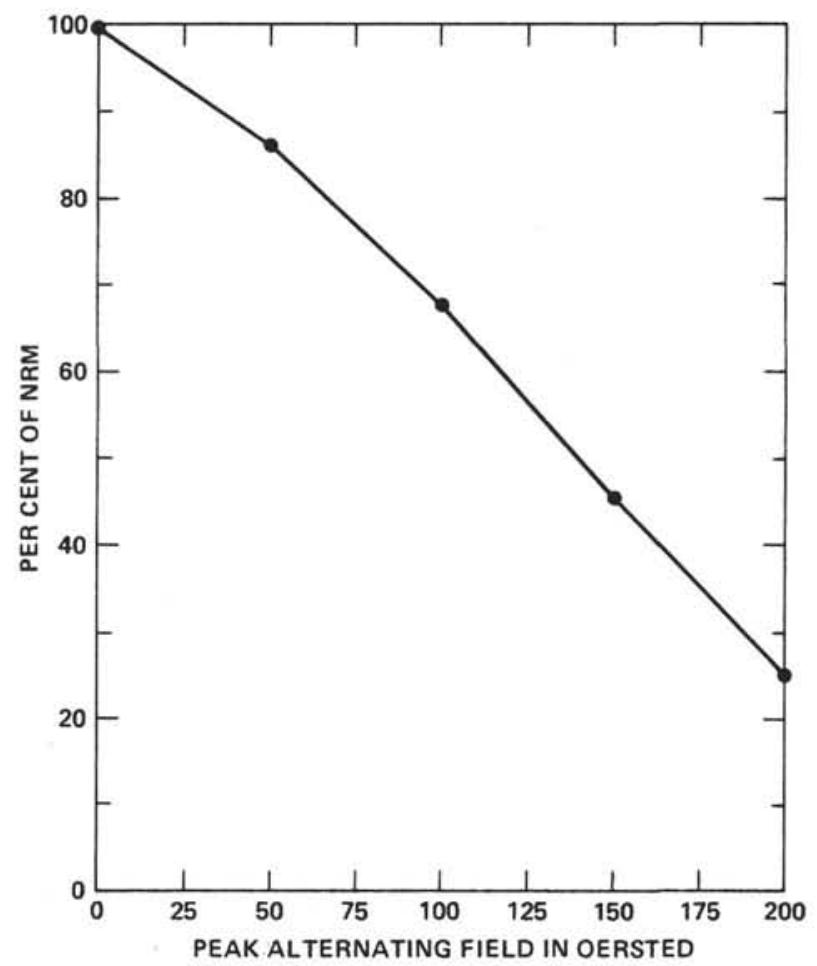

Figure 8. 3-18-6-6, depth $75-77 \mathrm{~cm}$. 


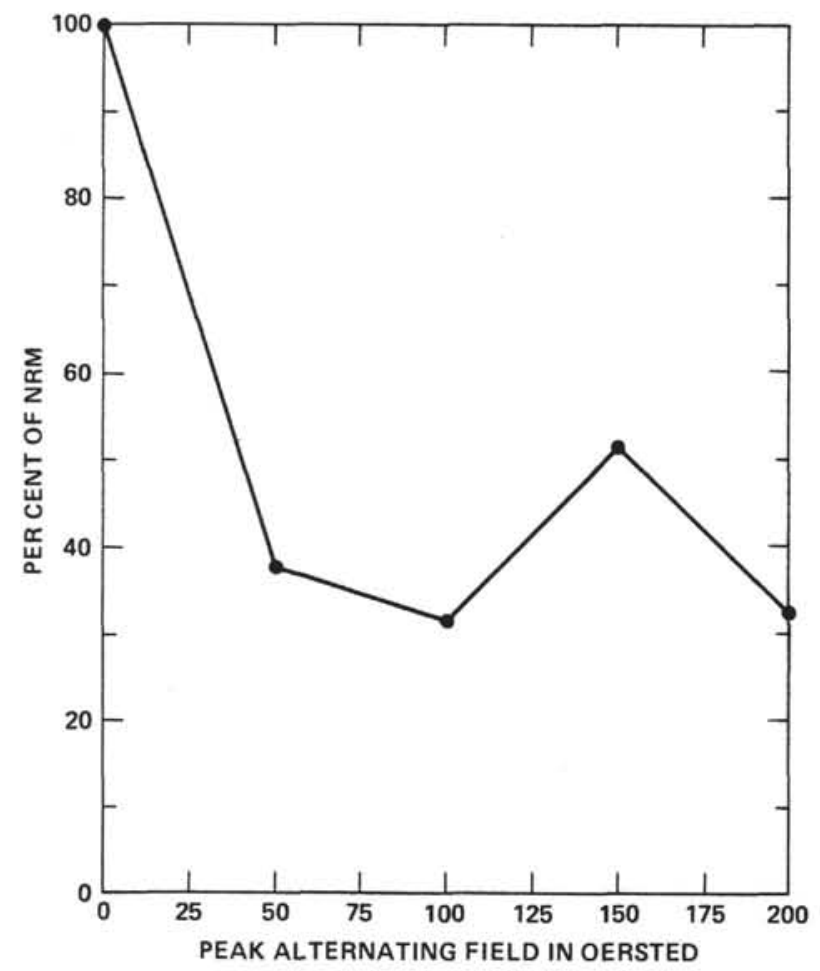

Figure 9. 3-19-1-2, depth 2-4 cm.

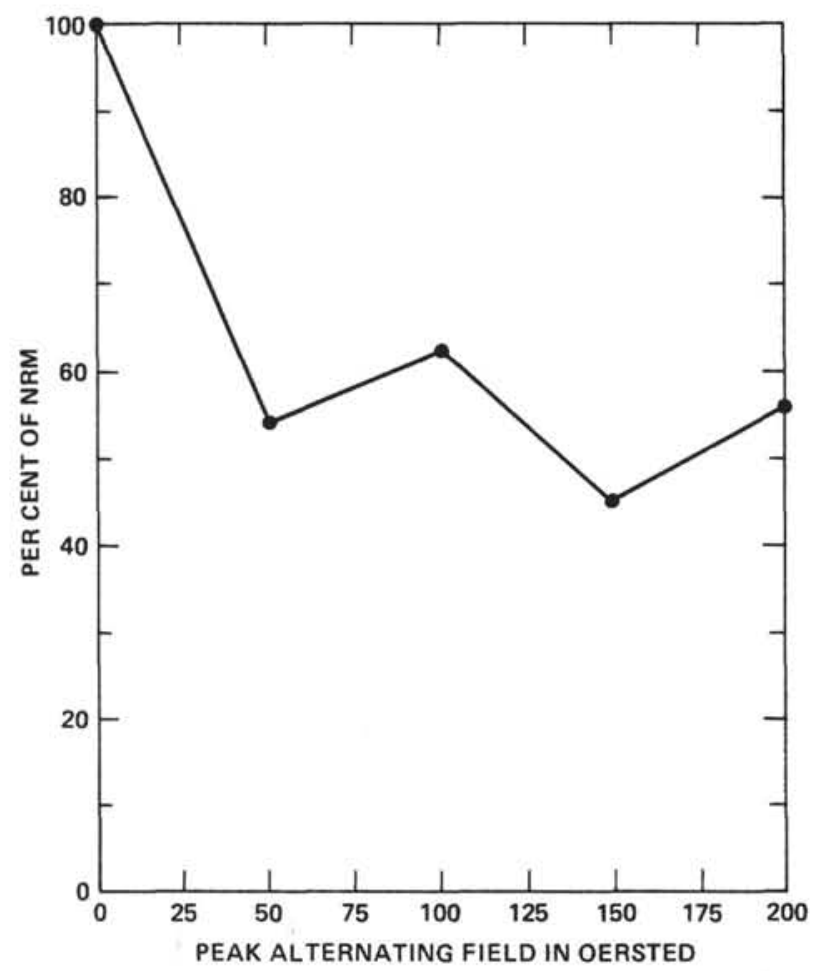

Figure 11. 3-20B-1-2, depth 7-9 cm.

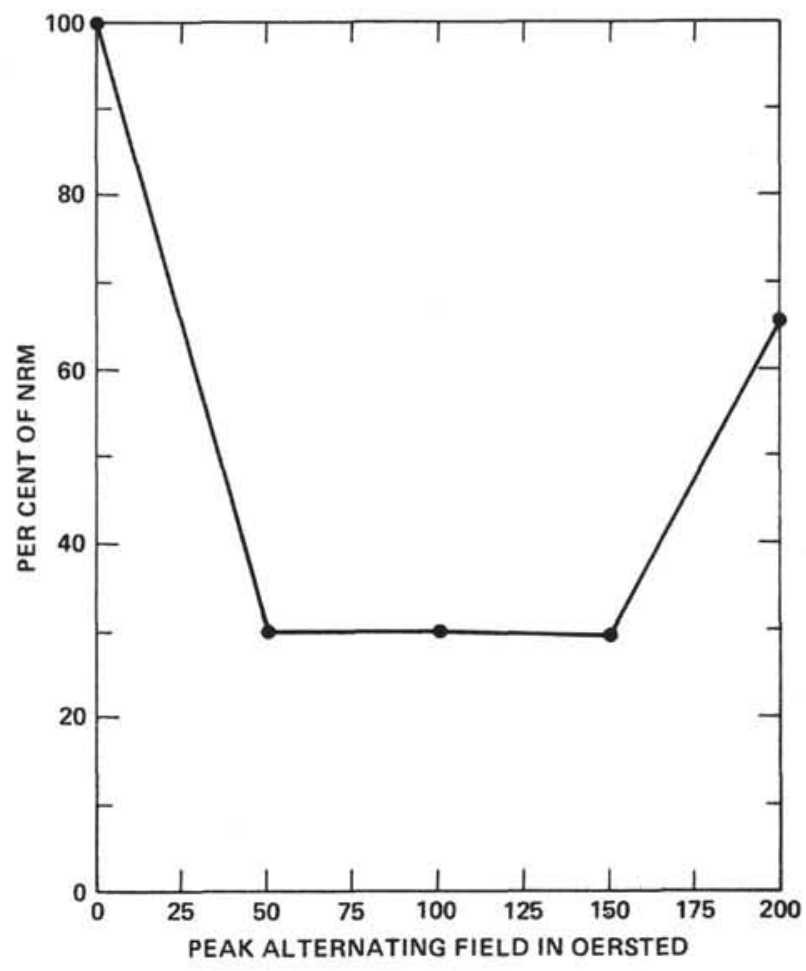

Figure 10. 3-20A-2-2, depth $50-52 \mathrm{~cm}$.

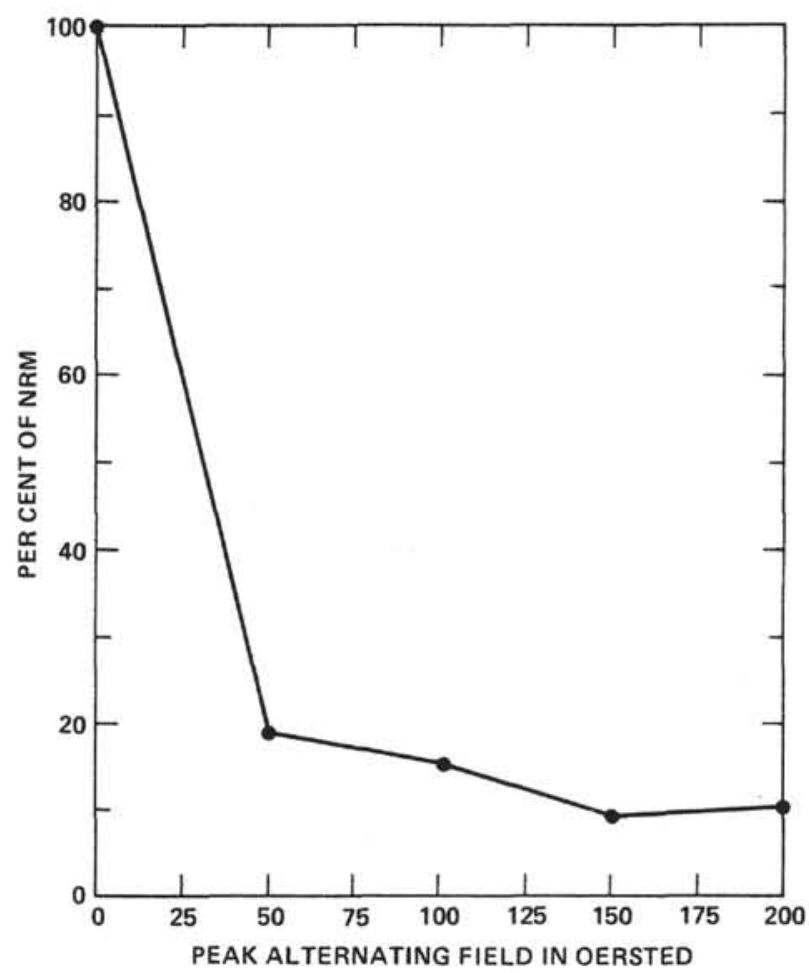

Figure 12. 3-20C-5-4, depth 60-62 cm. 


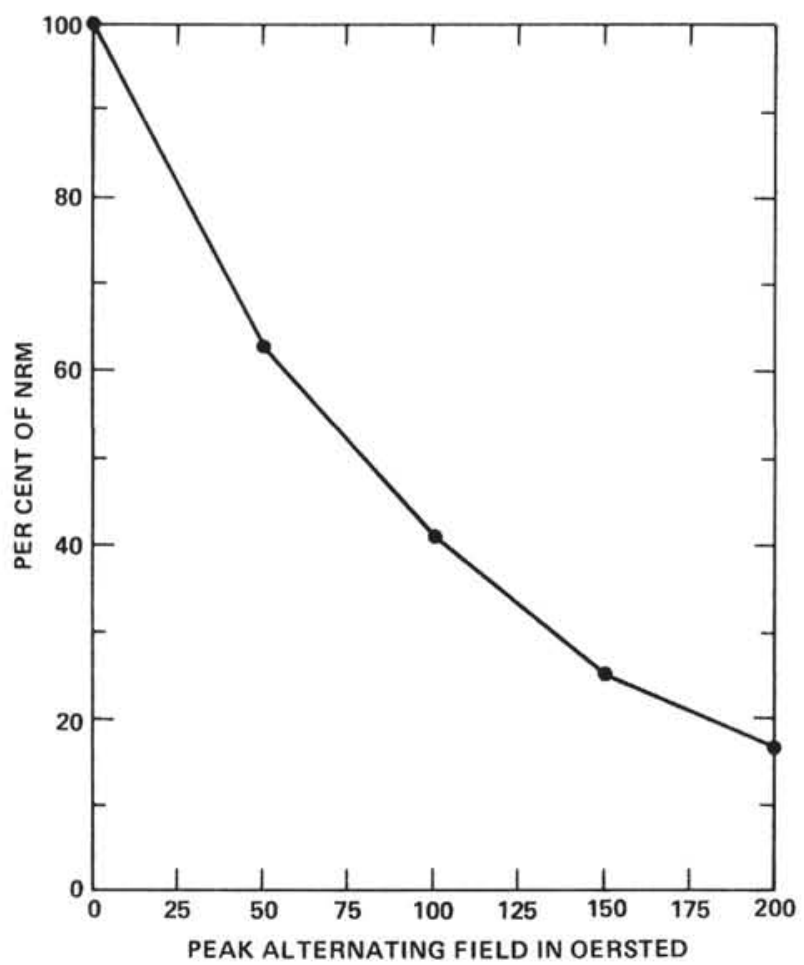

Figure 13. 3-21-6-6, depth 3-5 cm.

Figures 14 through 20

Magnetic Stratigraphies of Sites 14 through 20

Normal field direction is indicated in black; reversed field is striped. Actual sampled points are indicated by arrows. A scale of depth below the ocean floor in meters is shown at the left; ages are indicated to the right. All polarities are based on measurement after A-F demagnetization in 50 oersteds. 

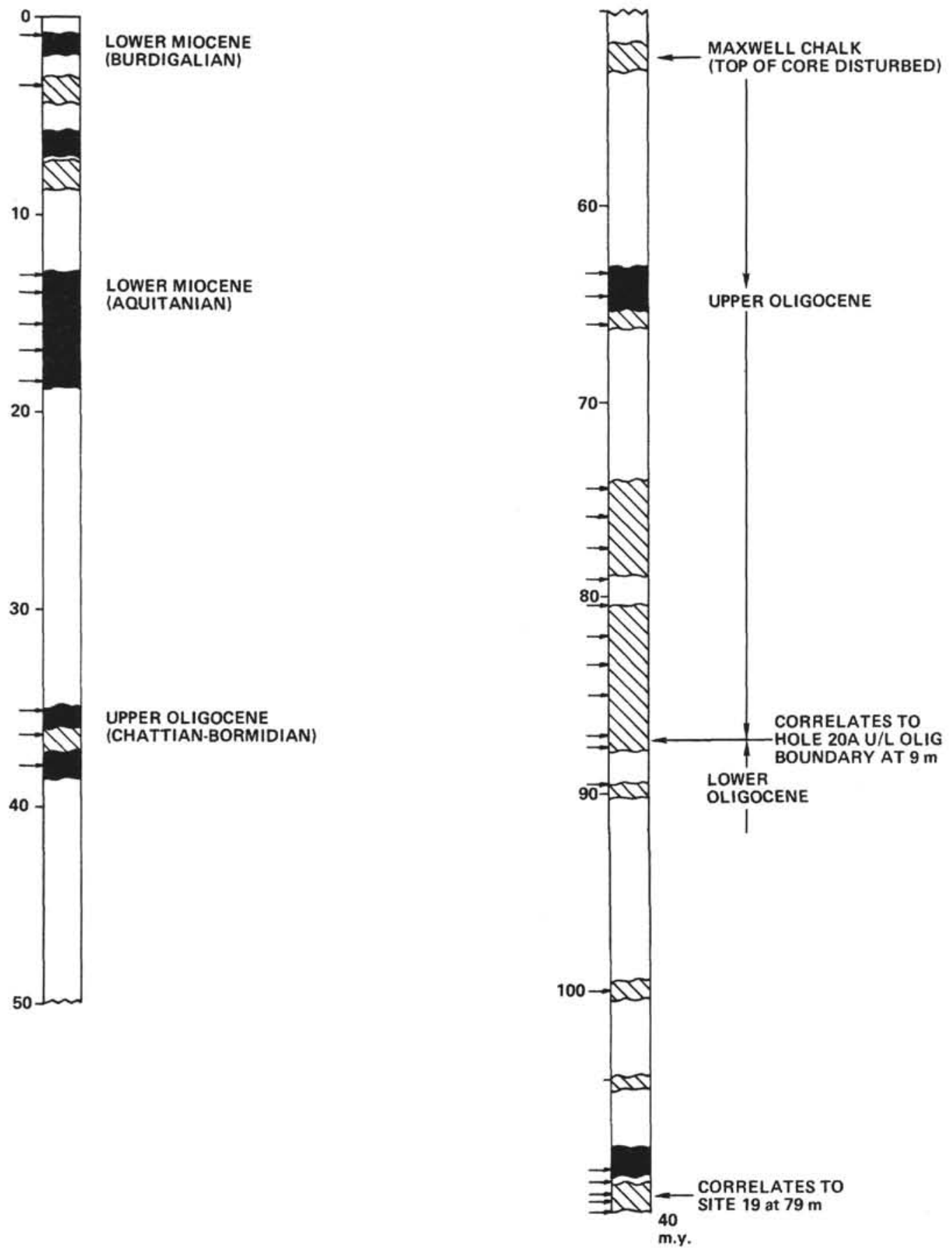

Figure 14. Magnetic Stratigraphy of Site 14. 

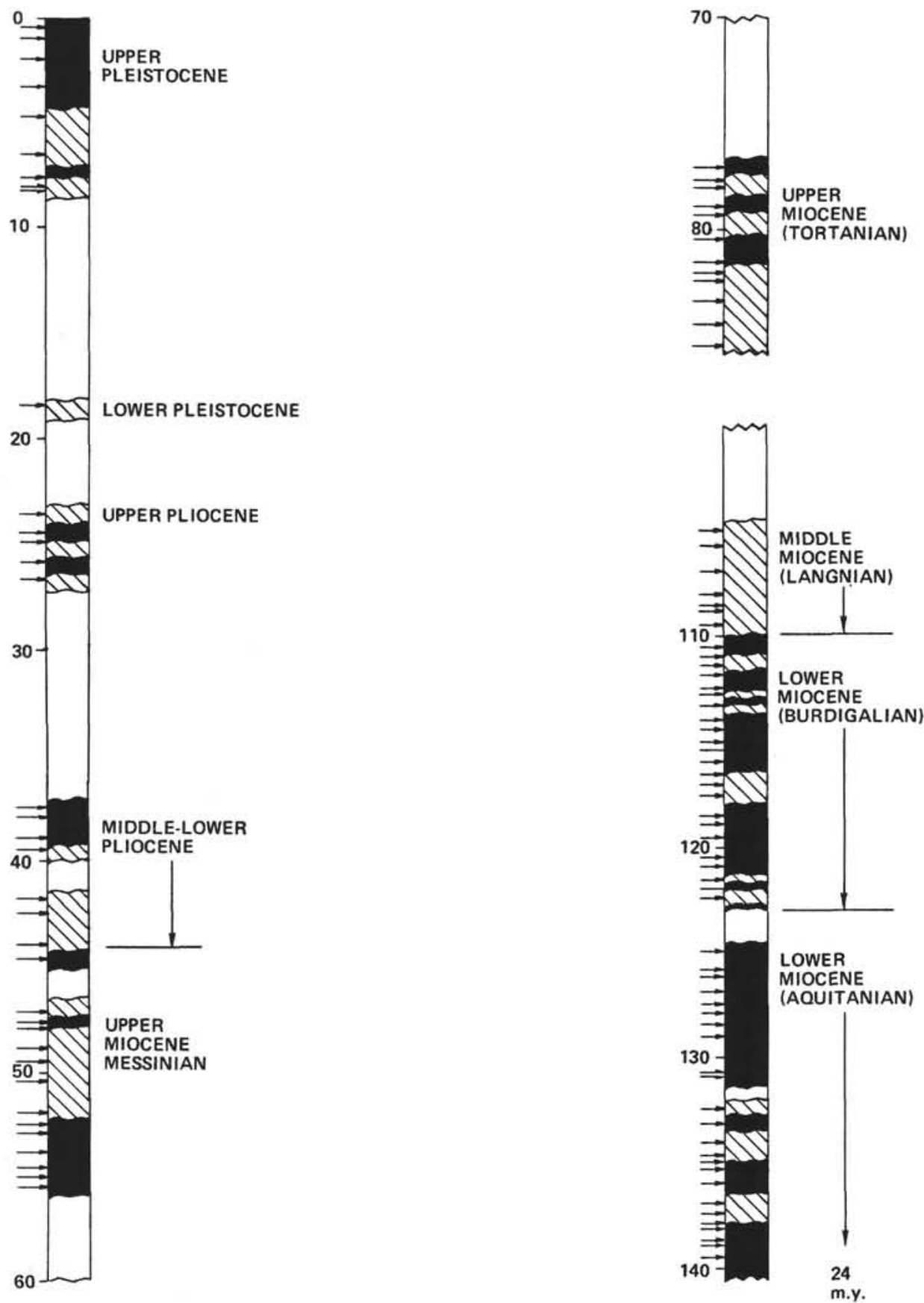

Figure 15. Magnetic Stratigraphy of Site 15. 


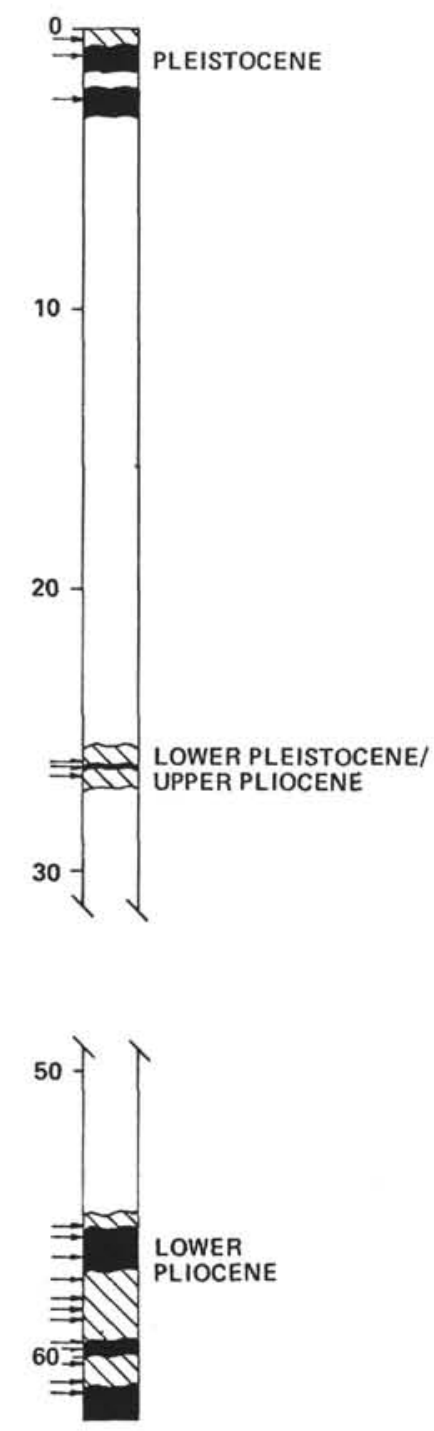

Figure 16. Magnetic Stratigraphy of Site 16. 

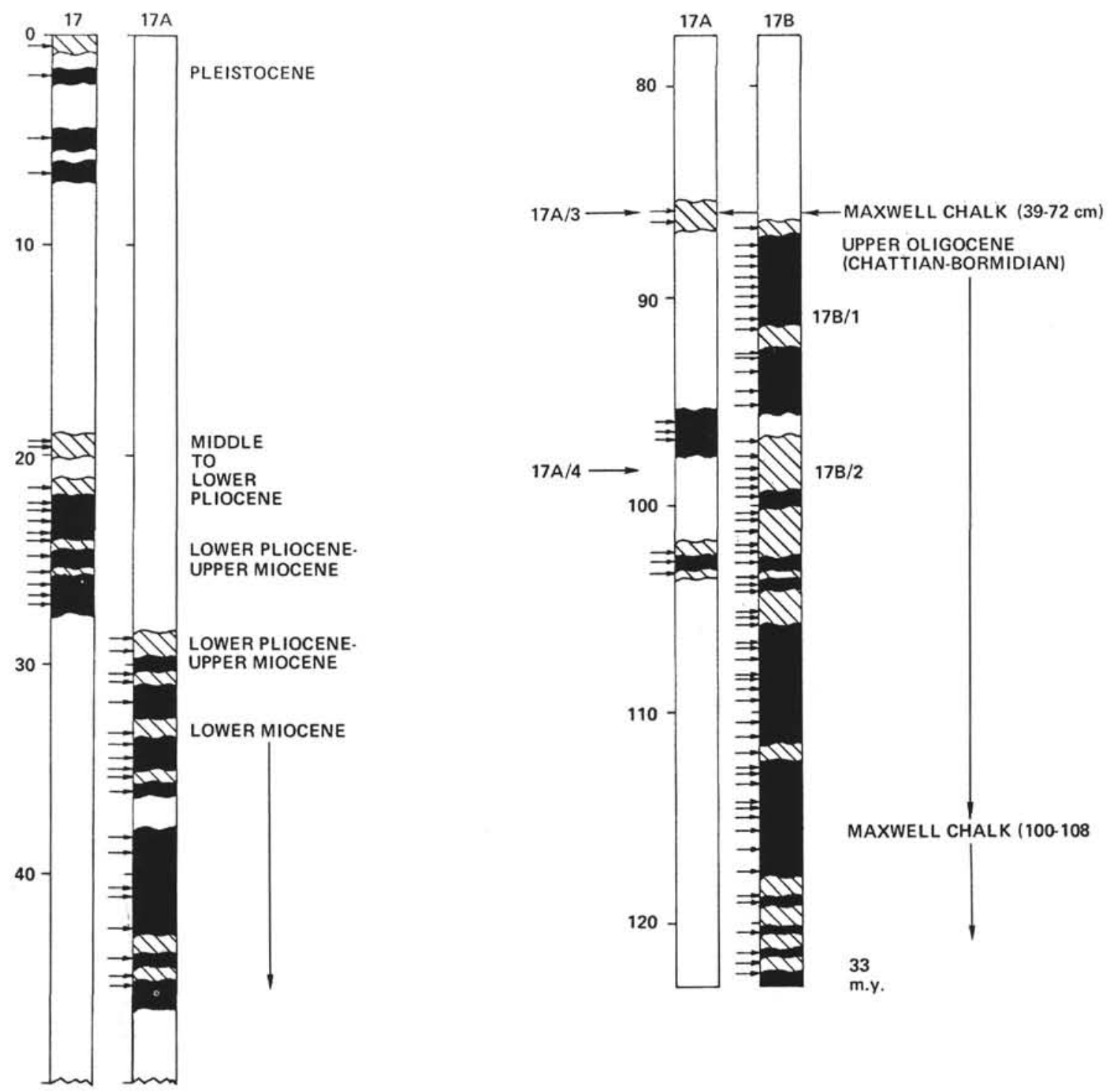

Figure 17. Magnetic Stratigraphy of Site 17. 


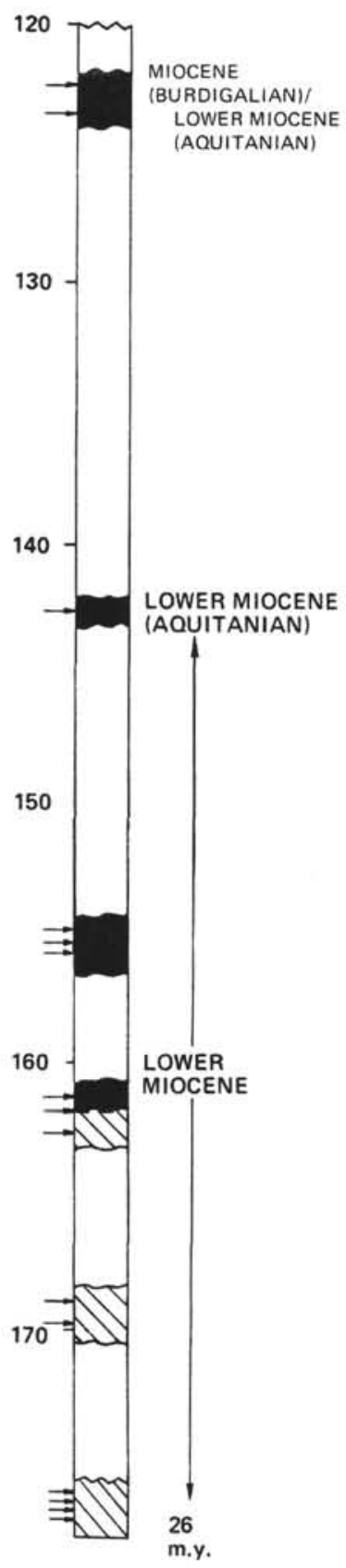

Figure 18. Magnetic Stratigraphy of Site 18.
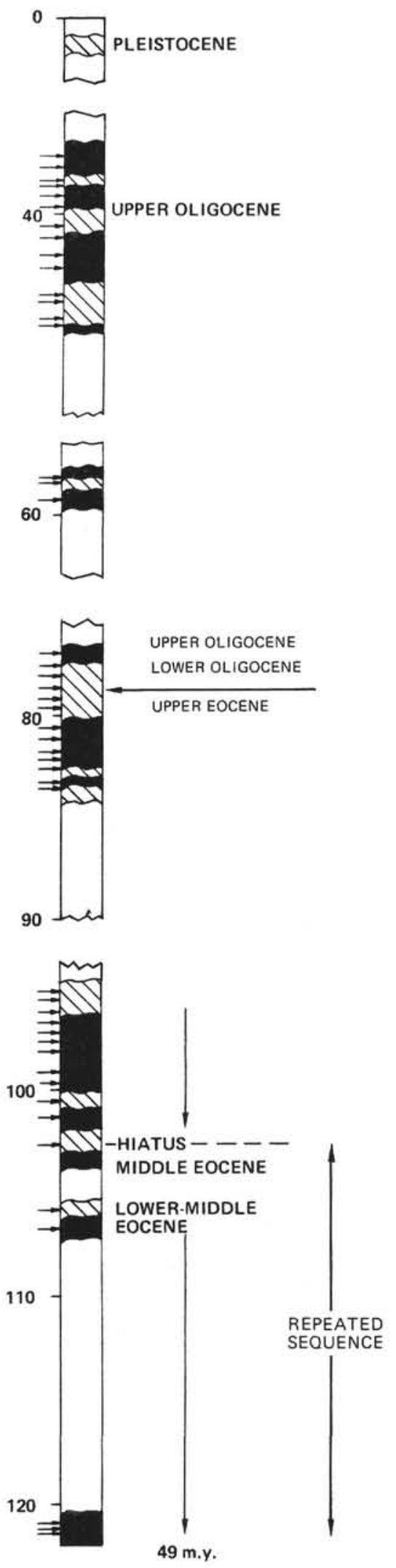

Figure 19. Magnetic Stratigraphy of Site 19. 


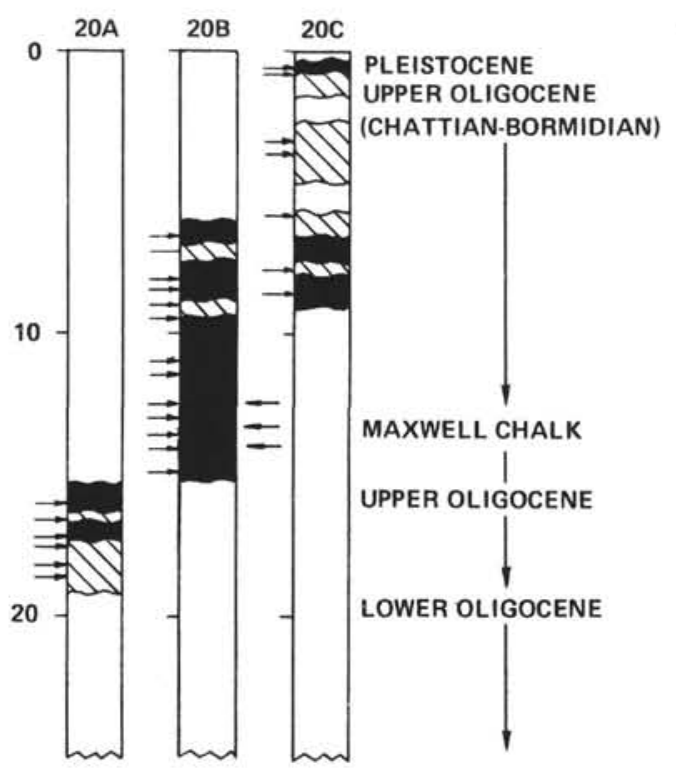

Figure 20A. Magnetic Stratigraphy of Site 20, 0-25 m. subbottom.

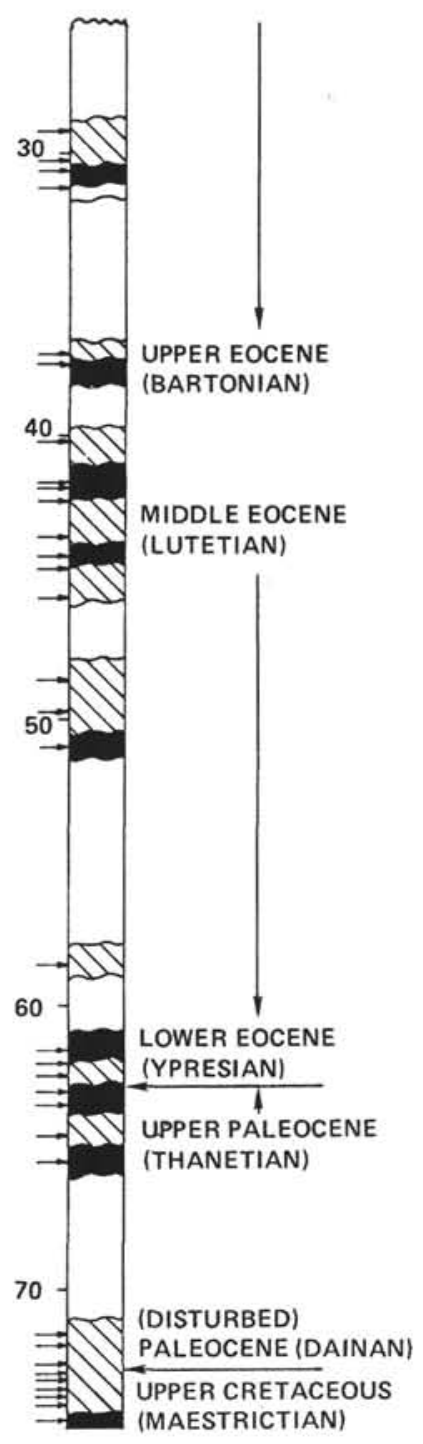

66 m.y.

Figure 20B. Magnetic Stratigraphy of Site 20, Hole 20C, $25 \mathrm{~m}-75 \mathrm{~m}$. subbottom. 